\title{
Predicting the Cavitating Marine Propeller Noise at Design Stage: a Deep Learning Based Approach
}

\author{
Leonardo Miglianti $^{\mathrm{a}}$, Francesca Cipollini ${ }^{\mathrm{b}}$, Luca Oneto ${ }^{\mathrm{c}}$, Giorgio Tani $^{\mathrm{a}}$, \\ Stefano Gaggero $^{\mathrm{a}}$, Andrea Coraddu ${ }^{\mathrm{d}, *}$, Michele Viviani ${ }^{\mathrm{a}}$ \\ ${ }^{a}$ DITEN - University of Genoa, Via Opera Pia 11A, 16145 Genoa, Italy \\ ${ }^{b}$ aizoOn Technology Consulting, Strada del Lionetto 6, 10146 Turin, Italy \\ ${ }^{c}$ DIBRIS - University of Genoa, Via Opera Pia 13, 16145 Genoa, Italy \\ ${ }^{d}$ NAOME - Strathclyde University, Glasgow G1 $1 X W, U K$
}

\begin{abstract}
The importance of reducing the noise impact of ships is being recognised worldwide. Consequently, the inclusion of this principle among the objectives and constraints of new designs is becoming a standard. For this reason, considerable attention is given to the propeller being often the dominant source of underwater radiated noise, especially when cavitation occurs, as it happens in most cases when a ship sails at design speed. The designers of quieter propulsion systems require the availability of predictive tools able to verify the compliance with noise requirements and to compare the effectiveness of different design solutions. In this context, tools able to provide a reliable estimate of propeller noise spectra based just on the information available during propeller design represent a fundamental tool to speed up the design process avoiding model scale tests. This work focuses on developing a tool able to predict the cavitating marine propeller generated noise spectra at design stage exploiting the most recent advances in Deep Learning, able to take advantage of both structured and unstructured data, and in hybrid modelling, able to exploit both data and physical knowledge about the problem. For this purpose authors will make use of a dataset collected by means of dedicated model scale measurements in a cavitation tunnel combined with the detailed flow characterisation obtainable by calculations
\end{abstract}

\footnotetext{
* Corresponding Author

Email addresses: leonardo.miglianti@edu.unige.it (Leonardo Miglianti), francesca.cipollini@aizoon.it (Francesca Cipollini), luca.oneto@unige.it (Luca Oneto), giorgio.tani@unige.it (Giorgio Tani), stefano.gaggero@unige.it (Stefano Gaggero), andrea.coraddu@strath.ac.uk (Andrea Coraddu), michele.viviani@unige.it (Michele Viviani)
} 
carried out with a Boundary Element Method. The performance of the proposed approaches are analysed considering different scenarios and different definitions of the input and output variable used during the modelisation.

Keywords: Marine Propeller, Cavitation Noise Prediction, Spectra Parametrization, Deep Learning, Hybrid Models.

\section{Introduction}

Anthropogenic sources of sound in the marine environment are commonly related to navigation activities. The main sources of noise on board a vessel are machinery and propellers. The latter is the first contributor to radiated noise in water and, in case of cavitation, the noise levels increase abruptly [1]. Noise pollution adversely affects marine animals, people on board, and impinges with the on board equipment like sonars. In this framework, international organisations and class societies started to emanate non-mandatory guidelines to reduce the noise emissions, as the adoption of the Marine Strategy Framework Directive of the European Union [2] or the International Maritime Organisation (IMO) guidelines [3].

Within the different noise mitigation strategies that can be adopted by the shipping industry, the analysis of design solutions aimed at the reduction of radiated noise for new ships is of utmost importance. Focusing on conventional propeller design, cavitation should be minimized to reduce radiated noise. This is commonly achieved by different strategies, whose effectiveness depends on the specific application. Non-exhaustive list includes: variation of main propeller characteristics, increase of the blade area, unloading of the blade tip, adoption of a proper skew distribution, modification of blade sectional profiles. In this framework, design by optimization may represent an effective approach to reduce cavitation and consequently noise, as shown in [4, 5. However, the reduction of cavitation is usually conflicting with efficiency maximization, therefore an optimum trade-off must be defined case by case. This task requires the availability of tools and procedures for the prediction of the vessel's radiated noise even at the design stage, to verify and compare the benefits of different design solutions. Focusing on propeller noise, Model Scale Tests (MSTs) in cavitation tunnels are traditionally considered the most reliable method for cavitation noise prediction. MSTs make use of a prototype of the propeller, which is tested reproducing the full scale loading conditions. This approach represents a valuable tool both for the final verification of the propeller design and for the comparison of few alternative solutions, although scale effects must be carefully taken 
into account to retrieve the full scale noise from MSTs. However, MSTs are time-consuming, and their inclusion in the early stages of the design procedure is unpractical.

In this context, the availability of a propeller noise predictive tool, based on the information available at the design stage, is of paramount importance to reach cost and time effectiveness.

A possible approach to address this problem is represented by the use of simplified Physical Models (PMs) combined with empirical relations based on available experimental data. These methods are based on simplified formulations of the propeller cavitation development and consequent noise. These formulations are typically derived from idealised cases and adapted to the complex problem of propeller action, by means of fitting variables. As an example, a formulation for the prediction of the characteristic frequency of noise generated by cavitating vortices is presented in [6]. Although this formulation succeeds in describing the behaviour of vortex noise, it may require case-by-case tuning, being based on a simple 2-dimensional vortex model. An empirical relation for the amplitude of vortex noise was presented in [7. A method for the prediction of tip vortex noise based on both methods and on a typical spectral shape, has been presented in 8 . However, as claimed by the authors, the method is not able to model the phenomenon considering all the possible dynamics, as for instance the interactions with other phenomena, if present. A simple empirical formulation for the noise generated by thruster propellers has been presented in [9]. This formulation provides a simple relation between the amplitude of noise and the area of sheet cavitation. Notwithstanding its lack of insight into the physics of a cavitating propeller, this method can be adopted also to predict the noise of open propellers with satisfactory results, as presented in [10].

In general, the functional form of these formulations is derived from the physical equations describing the phenomenon under some simplifying assumptions. The formulations are successively tweaked on available experimental data to adapt them to practical cases.

An attractive alternative consists of tackling the problem utilising Data Driven Models (DDMs), as proposed in present work. DDMs approximate the relations existing between some target quantities (the targets) and the available data on quantities and phenomena influencing the targets (the features). This is achieved through robust statistical inference procedures and data collected in previous experiments, including both features and targets, to make predictions about previously unseen cases. These methods do not need any a-priory knowledge about the mathematical expression governing the physical system. As a consequence, the DDMs can also model complex 
propeller cavitation patterns, including possible interactions between different phenomena, without the need to consider any simplifying assumptions.

DDMs have proven to be valuable instruments in many marine applications, such as fuel consumption and efficiency prediction or ship components condition-based maintenance via status prediction [11-14], or to determine the best propeller design given operational requirements and constraints [15]. The application of DDMs in the field of ship radiated noise is mostly limited to classification problems [16, 17, while there is a lack of work regarding their application to the problem of ship and propeller noise modelling. Recently [18] proposed an Artificial Neural Network approach for the prediction of the propeller cavitation noise given a number of design parameters and an extensive collection of noise samples from cavitation tunnel tests.

In the view of developing DDMs, the employment of MSTs to collect data by means of systematic investigations of propeller noise is very desirable. Nevertheless, also in the case of MSTs, collecting a very large set of test cases could require significant effort in terms of cost and time. Consequently, the available data is typically not as large as required. In this context, the capability of DDMs, which usually produce black-box (non-parametric) models, may be limited in terms of generality.

In order to improve the performance of the DDMs, the knowledge of the physical phenomena included in the PMs can be exploited. This idea is the basis of Hybrid Models (HMs), which are developed to take advantage of the best characteristics of both PMs and DDMs by combining them together. The application of HMs to the problem of propeller noise modelling based on data obtained through MSTs has been presented in [19] with satisfactory results.

Models developed in the aforementioned works consider as features only the main characteristics and functioning parameters of the propeller. However, these quantities are generally not sufficient to describe the whole phenomenon that a cavitating propeller undergoes. Actually, propeller noise depends on the whole propeller geometry, on the flow field and the resulting cavitation pattern, including the effects of non-uniform propeller inflow, as in realistic conditions. Furthermore, if the considered features include only global parameters, it is not possible to appreciate the effects of specific design solutions, which may be remarkable for custom designed propellers. With the purpose to address these limits, the approach presented in this work exploits as features the detailed results of unsteady hydrodynamic computations, carried out by means of an in-house developed Boundary Element Method (BEM) [4, 20 22]. These features provide an accurate characterisation of the hydrodynamic field of the propeller at an acceptable 
computational cost, implicitly including the effects of the complete propeller geometry.

The hydrodynamic quantities computed by the BEM (i.e.unsteady surface pressure distributions over the blades, time varying strength of the vortexes shed in the propeller wake) include highly structured data in the form of $2 \mathrm{D}$ and $3 \mathrm{D}$ tensors. In order to fully exploit all the information contained in these tensors, some advanced techniques coming from the world of Deep Learning [23] have been proposed. These techniques allow first to find a rich representation of the tensors by finding all the information describing the cavitation phenomena and then to exploit it to make an effective and sufficient prediction of the propeller cavitation noise.

These models have been compared with the conventional models previously presented in 19], where the 1D representation was manually selected based on the theories on cavitation noise. It is demonstrated that the advanced models outstand the performance of the conventional ones if a high dimensional and complex dataset is considered. On the contrary, if a low dimensional dataset composed only by simple variables is taken into account, the performance of the advanced model is close to that of the more conventional models. As a result, the proposed approach is proven to bring significant improvements with respect to more traditional techniques if a complex set of features is provided.

Furthermore, with respect to [19], the whole feature set has been rearranged in order to include only data easily obtainable at the early stage of propeller design. Different combinations of input variables and targets have been considered, allowing to analyse the relative merits of each.

The paper is organised as follows. Section 2 describes the experimental test cases, the definition of the targets and the different feature sets considered. Section 3 reports the adopted PMs and the proposed DDMs and HMs. The rerults of PMs, DDMs, and HMs in the two scenarios described above are reported in Section 4. Finally, Section 5 concludes the paper. For increasing the readability of the paper Table 1 reports the acronyms exploited in the paper. Due to confidentiality issues, sensitive data like propeller geometry and loading conditions will be omitted or altered by means of appropriate reference values kept constant throughout the whole article.

\section{Problem definition}

The cavitating propeller noise depends on several aspects concerning the characteristics of the propeller and its functioning conditions. Present work 


\begin{tabular}{|l|l|}
\hline \hline ADDM & Advanced Data Driven Model \\
BEM & Boundary Element Method \\
BSRA & British Ship Research Association \\
CDDM & Conventional Data Driven Model \\
CPP & Controllable Pitch Propeller \\
DDDM & Deep Data Driven Model \\
DDM & Data Driven Model \\
ELM & Extreme Learning Machine \\
ERM & Empirical Risk Minimisation \\
FS & Feature Set \\
IMO & International Maritime Organisation \\
ITTC & International Towing Tank Conference \\
KRLS & Kernel Regularised Least Square \\
MAE & Mean Absolute Error \\
MAPE & Mean Absolute Percentage Error \\
MST & Model Scale test \\
NSP & Noise Spectrum Parametrization \\
OTO & One Third Octave \\
PM & Physical Model \\
PPMCC & Pearson Product Moment Correlation Coefficient \\
RNL & Radiated Noise Level \\
SDDM & Shallow Data Driven Model \\
SNR & Signal to Noise Ratio \\
SPL & Sound Pressure Level \\
TF & Transfer Function \\
UNIGE & University of Genoa \\
\hline
\end{tabular}

Table 1: Acronyms exploited in the paper.

aims to model the unknown relation existing among some input variables describing the propeller (the features) and the corresponding output variables representing the radiated noise (the targets). This relation is to be learned from the previous observations of the phenomenon (the available dataset). The features should include all the possible quantities which could influence the outcomes of the modelled phenomenon; the targets represent this outcome. The final aim of the model is to predict it for previously unseen cases.

The observations of the phenomenon are provided by model scale tests carried out at the cavitation tunnel, for which the input features and the targets are known from computations or direct measurements. Both features and targets are used by the procedures employed to build the models.

The next sections describe the different sets of features and targets which compose this dataset; in particular, five different sets of input features have been determined:

- Feature Set 1 (FS1), consisting in a set of scalar parameters providing a global description of the propeller and its functioning;

- Feature Set 2 (FS2), consisting in the complete tensor of resulting 
angle of attack values;

- Feature Set 3 (FS3), consisting in the complete tensor of inflow wake;

- Feature Set 4 (FS4), consisting in the complete tensor of pressure coefficients on the blades;

- Feature Set 5 (FS5), consisting in the complete tensor of blade circulation.

Moreover, five different parametrizations of the noise signals have been determined as output features in order to allow an easy identification of the error for each proposed model:

- Noise Spectra Parametrization 1 (NSP1), consisting in the noise frequency and level of the central peak characterizing the spectra;

- Noise Spectra Parametrization 3 (NSP2), consisting in the series of relevant parameters characterizing the spectra proposed in [19];

- Noise Spectra Parametrization 2 (NSP3), consisting in a new series of relevant parameters characterizing the spectra;

- Noise Spectra Parametrization 4 (NSP4), consisting in the source levels in one-third octave band representation;

- Noise Spectra Parametrization 5 (NSP5), consisting in the rsdited noise levels in one-third octave band representation.

\subsection{Model Scale Tests}

The noise measurements campaign has been carried out, according to the International Towing Tank Conference (ITTC) model scale noise measurement guidelines [24, at the cavitation tunnel of the University of Genoa.

The facility, described in detail in [25], is a closed-circuit water tunnel with adjustable pressure and equipped with a square test section measuring $0.57 \times 0.57 \times 2 \mathrm{~m}$. An impeller is used to obtain a specific flow rate in the test section, while an independent engine runs the tested propeller at the required shaft rate. The propeller thrust, torque and revolution rate are measured by a Kempf \& Remmers H39 dynamometer. Specific values of the advance ratio $J$, as defined in Eq. (1), are obtained by adequately setting the flow rate and the propeller shaft rate, while adjusting the tunnel pressure it is possible to modify the cavitation number $\sigma$ defined in Eq. (2)

$$
\begin{aligned}
J & =\frac{V_{a}}{n D}, \\
\sigma & =\frac{p_{\infty}-p_{v}}{0.5 \rho V_{r e f}^{2}},
\end{aligned}
$$

where $V_{a}$ is the advance speed, $n$ is the revolution rate, $D$ is the propeller diameter, $p_{\infty}$ is the undisturbed pressure, i.e. the static head at a certain 
location on propeller disk, $p_{v}$ is the vapour pressure, $\rho$ is the water density and $V_{\text {ref }}$ is a reference speed. Based on the choice of the reference speed and the reference point for computing $p_{\infty}$, different definitions of the cavitation number may be obtained. In most cases, the cavitation number $\sigma_{n}$, based on the peripheral tip speed $\left(V_{\text {ref }}=n D\right)$ is used, considering the centre disk as the reference point for the static head.

The advance ratio and the cavitation number allow defining the propeller loading conditions in terms of kinematic and cavitation conditions, and are usually set in such a way to achieve a similarity condition with the full-scale propeller. The equivalence of these parameters allows reproducing the propeller functioning conditions in terms of average parameters. However, the real propeller operates behind a ship hull, hence in a three-dimensional disturbed flow field. This field is usually given as the distribution of the local velocity components in polar coordinate in correspondence to the propeller disk. Its characteristics depend on the hull geometry and shaftline configuration. In general, a significant reduction of the axial velocity is visible in the upper part of the disk, caused by the boundary layer of the hull and/or the wake of appendages.

In the case of a twin screw vessel, this velocity deduction is rather moderate whereas a significant in-plane component is present, directed mainly upward. Several techniques exist to reproduce the wake of a ship in a cavitation tunnel [26]. Those used at UNIGE cavitation tunnel include: a small simulacrum of the aft part of a ship and its shaft-line, wire screens, shaft appendages and shaft inclination.

Radiated noise is measured by one, or more, hydrophone(s) placed in the tunnel test section and it is acquired as pressure time traces. Since most of the regulations and requirements concerning the acoustic signature of ships are given in terms of frequency spectra, the results of model scale noise measurements are usually given in terms of noise spectra, obtained by the post-processing described hereafter.

Noise spectra in narrowband representation are computed with a Fast Fourier Transform, using Welch's method of averaging modified spectrograms [27. Spectra in One Third Octave (OTO) band are obtained by integrating the power spectral density on the relevant bands, or by computing the root mean square of the signal filtered on the same bands. This first processing provides narrowband and OTO band spectra of the total received noise $\mathrm{SPL}_{t}$ measured by the sensor.

This noise is generally corrupted by the background noise of the facility $\mathrm{SPL}_{b}$, such as tunnel machinery, shaft gear, flow noise and by the propeller non-cavitating noise. As an example, it is depicted in Figure 1, the com- 
parison between the total noise and the background noise experimentally measured, for a loading condition ${ }^{1}$, it is evident the cavitation noise contribution on the total noise, for the whole frequency range.

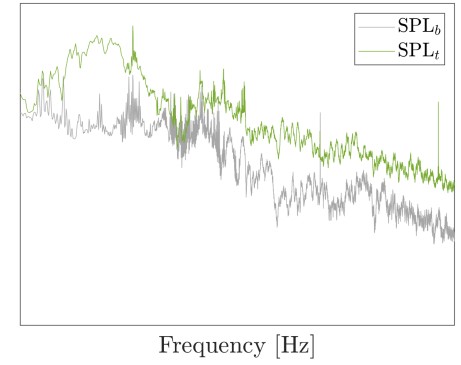

(a) Narrow band representation.

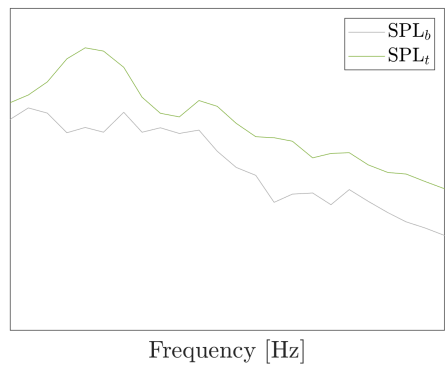

(b) OTO bands representation.

Figure 1: Total and background noise measure for the propeller P2 at design pitch, in a mid-loaded condition with $\tilde{\sigma}_{n}=0.75$.

As a consequence, a background noise correction is applied to extract the cavitation contribution. In order to apply this correction, the background noise is measured for each loading condition, reproducing the same flow speed, shaft rate and cavitation number of the propeller tests, but replacing the propeller with a dummy hub. Thereafter, provided that a sufficient Signal-to-Noise Ratio (SNR) is obtained, the Sound Pressure Levels (SPLs) referred to the cavitation noise $\mathrm{SPL}_{n}$ are obtained by logarithmic subtraction of the background noise from the total noise, as specified in [26].

Distance normalisation is required to account for the power decay due to the radial distance $r$ from the acoustic source to the hydrophone. Following the hypothesis of spherical spreading loss (free-field condition), the Radiated Noise Levels (RNLs) referred to the $\mathrm{r}_{r e f}$ distance of $1 \mathrm{~m}$ from the propeller disk centre are

$$
\mathrm{RNL}_{1 / 3}=\mathrm{SPL}_{n}+20 \log _{10}\left(\frac{\mathrm{r}}{\mathrm{r}_{\text {ref }}}\right)
$$

The unit of measure is, in one-third octave band levels, $\mathrm{dB}$ re $1 \mu \mathrm{Pa}^{2} \mathrm{~m}^{2}$. The processing just described does not take into account the influence of reverberation and other effects related to the confined environment in which measurements are carried out. These phenomena may be schematised by

\footnotetext{
${ }^{1}$ The cavitation number $\tilde{\sigma}_{n}$ is divided by a reference value for confidentiality reasons.
} 
the Transfer Function (TF) of the facility, which can be measured with the procedure described in [28, 29]. The propeller Source strength Levels (SL) are then computed as

$$
\mathrm{SL}_{1 / 3}=\mathrm{SPL}_{n}-\mathrm{TF},
$$

the unit of measure is still $\mathrm{dB}$ re $1 \mu \mathrm{Pa}^{2} \mathrm{~m}^{2}$.

The outcome of such computations is visible in Figure 2, the loading conditions are characterised by the same thrust coefficient but different cavitation numbers. The transfer function correction is dependent upon the frequency, for this the shape and levels of the noise spectra are different between RNLs and SLs.

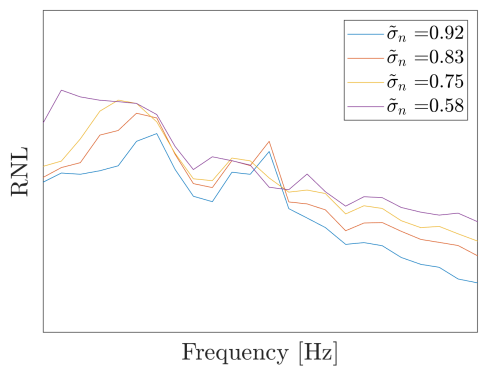

(a) Radiated net noise in OTO bands.

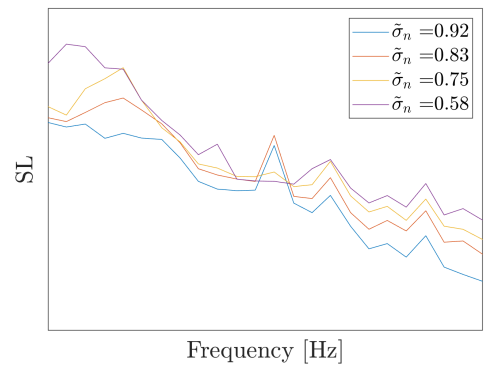

(b) Transferred noise in OTO bands.

Figure 2: Cavitation noise comparisons for the propeller P2 at design pitch, in various loading conditions.

\subsubsection{Test Cases}

For present work, MSTs have been carried out for three Controllable Pitch Propellers (CPPs), considering different pitch settings and wake configurations, as summarised in Tables 2 and 3 . The adopted wake fields are shown, in Figure 3, in terms of distributions of the axial wake fractions $w$, where $(1-w)=V_{a} / V$, being $V$ the undisturbed flow velocity. In these plots, $R$ is the propeller radius while $y$ and $z$ are the transverse and vertical coordinate in the propeller reference system. All these wake fields are typical examples of wakes of twin screw ships: W1 and W3 show the larger peaks, W4 is narrower and less decelerated, W6 is quite similar to the undisturbed flow except for the presence of a small region of decelerated flow at outer radii. Lastly, also the cases of uniform axial wake field (W2 and W5) have been considered. The little disturbance visible for W2, W4, W5 and W6 


\begin{tabular}{|l|c|c|c|}
\hline \hline Propeller & P1 & P2 & P3 \\
\hline \hline Type & CPP & CPP & CPP \\
Diameter & $0.25 m$ & $0.25 m$ & $0.2639 m$ \\
Number of blades & 5 & 5 & 5 \\
BAR & 0.755 & 0.612 & 0.6549 \\
Direction of rotation & Right & Right & Right \\
Design pitch ratio at 0.7R & 1.385 & 1.156 & 1.440 \\
Reduced $\left(-3^{\circ}\right)$ pitch ratio at 0.7R & 1.229 & 1.013 & - \\
Reduced $\left(-5^{\circ}\right)$ pitch ratio at 0.7R & - & 0.938 & - \\
Reduced $\left(-6^{\circ}\right)$ pitch ratio at 0.7R & 1.082 & - & - \\
Incremented $\left(+2^{\circ}\right)$ pitch ratio at $0.7 \mathrm{R}$ & - & 1.256 & - \\
Shaft inclination & $6.8^{\circ}$ & $2.5^{\circ}$ & $6.4^{\circ}$ \\
\hline \hline
\end{tabular}

Table 2: The model propellers characteristics.

near the hub is given by the vortical flow shed by the dynamometer shaft; the influence of these structures on the cavitation is negligible.

\begin{tabular}{l}
\hline \hline Propeller \\
\hline Pitch
\end{tabular}

Table 3: Propeller setups.

Tests have been carried out with a Reynolds number ranging from $1.25 \times 10^{6}$ to $1.6 \times 10^{6}$ and an advance ratio from 0.6 to 1.2 . The loading conditions, for which noise samples are collected, have been defined to provide an exhaustive characterisation of cavitation noise, including many different phenomena. However, in the present work the attention is focused only on the loading conditions for which the most common cavitation types, namely tip vortex and suction side sheet cavitation, were dominant. As a result, the final dataset is composed by 258 samples, each sample corresponding to a row of the dataset.

\subsection{Features definition: integral quantities}

Selected features should provide complete information on the main aspects influencing the noise generated by a cavitating propeller. To a first approximation, this is mostly influenced by the propeller geometry, its functioning conditions and the quality of the inflow wake. The more detailed 


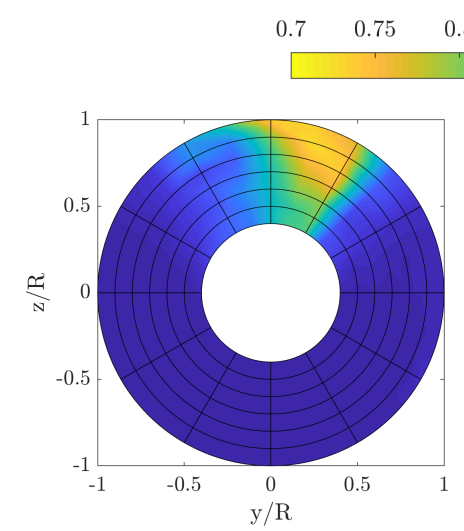

(a) Wake 1.

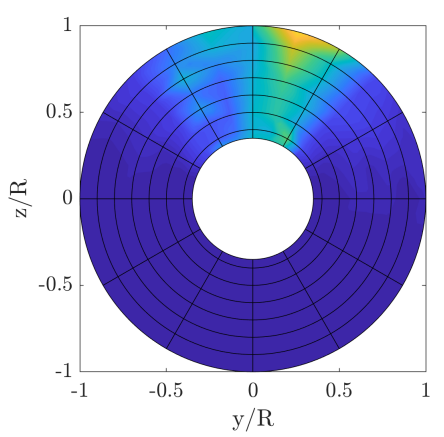

(c) Wake 3.

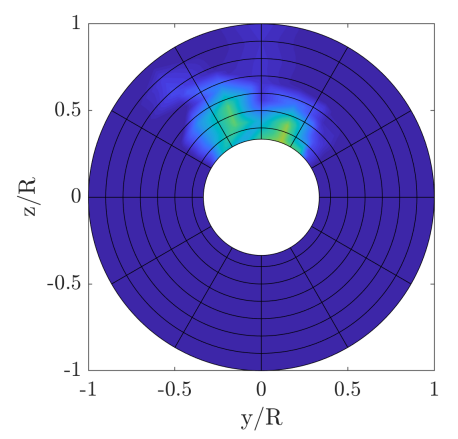

(e) Wake 5 .

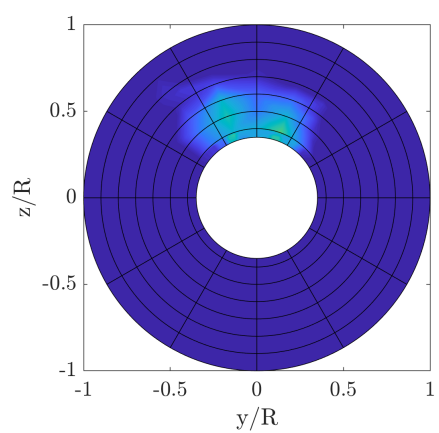

(b) Wake 2 .

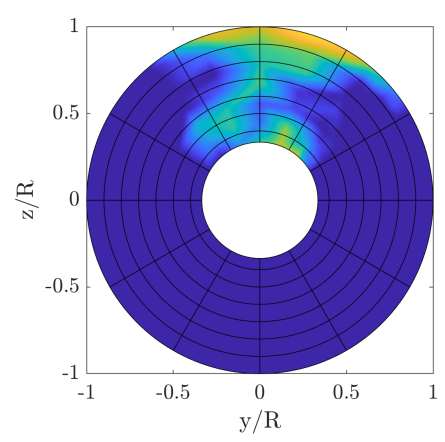

(d) Wake 4 .

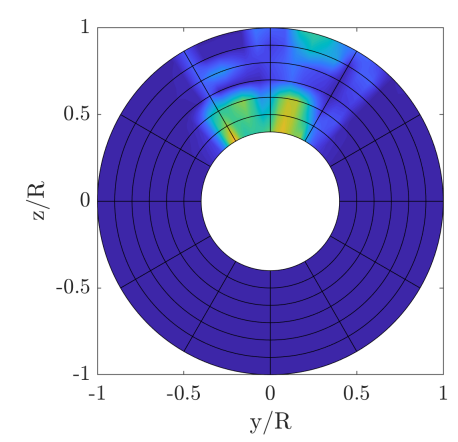

(f) Wake 6 .

Figure 3: Axial wake $(1-w)$ for the P1, the P2, and P3. 
information is available on these aspects, the better should perform the model.

The first proposed feature set, called FS1, consists of a set of parameters providing a global description of these aspects. This feature set is very similar to the one proposed in [19], having eliminated the features describing cavitation inception. The authors decided to remove these features since cavitation inception data are available at the design stage only using numerical computations characterised by a significant complexity and computational demand. Aiming to develop a predictive tool useful also during the preliminary design of a propeller, only quantities that can be retrieved at the design stage, using the kind of tools typically used by propeller designers, have been considered.

The FS1 features may be divided according to the following categories: propeller geometry, propeller operational conditions, and propeller inflow as summarised in Table 4 .

The propeller geometry is described through typical non-dimensional parameters such as the blade area ratio (BAR), the pitch ratio and others. The propeller diameter is considered as well, to provide dimensional information about the model propeller.

The operational conditions have been defined by the pair $\left(J, \sigma_{n}\right)$, hence the advance ratio and the cavitation number based on the tip peripheral speed are given by definition. The other quantities corresponding to these operational conditions (e.g. propeller thrust, torque and efficiency) have been included deriving them from the numerical computations using the Boundary Element Method or simple calculations based on the known relations among these parameters. Besides, direct measurements of these quantities are available from the MSTs, but are not considered in the feature sets for the previously mentioned reasons. Alternative definitions of the cavitation number have been considered, namely $\sigma_{v}$, based on the advance speed, and $\sigma_{t i p}$, that is the same as $\sigma_{n}$, but with the static head computed with respect to the top dead centre.

In addition to all these dimensionless parameters, dimensional quantities defining the functioning conditions, such as the advance speed, the revolution rate and the static pressure, are considered as well.

The last category of features included in FS1 accounts for the characteristics of the propeller inflow. Besides, also the geometric angle of attack of the blades is considered, which results from the combination of the inflow wake, the shaft inclination and the propeller pitch.

The hull wake field is usually known during propeller design from previous towing tank measurements or numerical calculations, hence it can be 
considered as a readily available input data for propeller designers. The wake fields described in Section 2.1 consist of distributions of the axial wake fraction on the propeller disk, given in polar coordinate, as shown in Figure 3. However, in the feature set FS1 only some parameters, providing an approximate description of these distributions, were considered.

The definition of these parameters is similar to the British Ship Research Association (BSRA) wake criteria given by [30], and it is based on the wake distribution in the tangential direction for the two radial sections corresponding to $r / R=0.7$ and $r / R=0.9$, as exemplified in Figure 4. In particular, the features are

- The left and right gradients, which are the maximum and minimum derivatives of the wake with respect to the angular blade position, computed at a given radial position.

- The wake width, which is the angular sector where the wake fraction is greater than 0.05 (i.e. where the axial velocity on the propeller plane is reduced at least of the $5 \%$ ).

- The wake depth, which is the maximum value of the wake fraction for a given radial position.

The shaft bracket produces the little decelerated peak visible at around $-30^{\circ}$ and it is neglected in the computation of these features because from experimental evidence it has only a minor effect on cavitation. The angle of

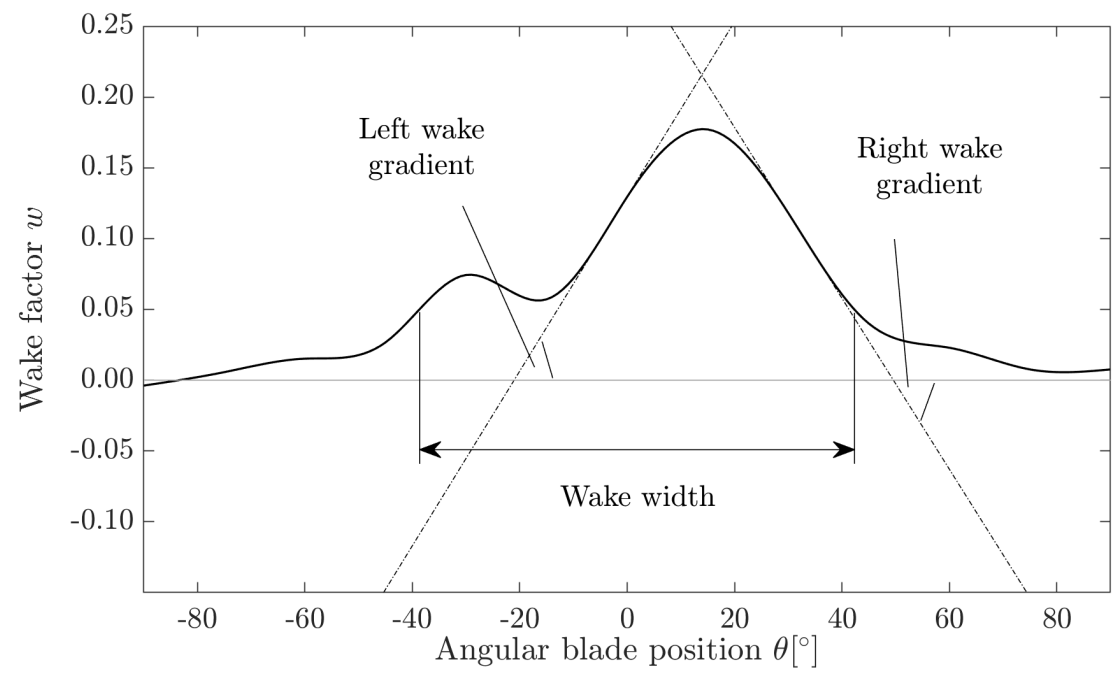

Figure 4: Wake parameters. 
attack is the difference between the advance angle $\beta$ of a blade section and the local pitch angle $\Phi$. The angle $\beta$ is determined by Eq. (5), including the effect of the axial wake field and the vertical velocity induced by the shaft inclination but neglecting the self-induced velocity field produced by the functioning of the propeller itself.

$$
\tan \beta(r, \theta)=\frac{V_{a}(1-w)}{\pi \cdot D \cdot n \cdot \frac{r}{R}+V_{a} \cdot \sin \left(\alpha_{s}\right) \cdot \sin (\theta)},
$$

where $\alpha_{s}$ is the shaft angle and $\theta$ is the blade angular position. The geometric angle of attack $\alpha_{G}$ is given by

$$
\alpha_{G}(r, \theta)=\Phi(r)-\beta(r, \theta) .
$$

As a result, a distribution of the angle of attack in polar coordinate is obtained, analogous to the distribution of the wake fraction. Also, in this case, the whole distribution is not considered in FS1, as the authors selected an approach analogous to that used for the wake.

It is worth noting that the features enlisted in Table 4 as FS0 are those that are directly employed in the PMs. Instead, $\mathrm{FSO}^{2}$ are variables that have been exploited to calculate other features for the PMs, as discussed in Section 3.2 .

In summary, FS1 is a set of scalar parameters, describing the average functioning of the propeller, plus an approximate indication about the nonstationary functioning of the blades given by the descriptors of the wake field and of the angle of attack. Accordingly, these parameters allow modeling the global inclination of the propeller to cavitation, hence noise, as confirmed by the use of some of these quantities also in many simplified methods for the preliminary analysis of propeller cavitation, as the well-known methods from [31] and [32. However, to improve the performance of the models, allowing to take into account also the effects of advanced design solutions, further features have been considered, as explained in the following section.

\subsection{Features definition: flow field quantities}

Accurate analyses of propeller cavitation require detailed information about the propeller inflow, the hydrodynamic pressure distribution on the blades, and the strength of the vorticity generated by the blades and shed in the wake. Only by the knowledge of these quantities it is possible to discriminate between different cavitation dynamics or to assess the effects of specific design solutions. For this reason, the authors decided to include 
this information in the feature set used to build the model, then moving to more sophisticated machine learning tools.

These new features consist in the complete wake field (and the resulting angle of attack), in the unsteady pressure distribution on propeller blades and in the circulation (i.e vortex strength) shed in the wake.

An approximate description of the wake field and angle of attack was already present in FS1. In light of using a more detailed description of the problem, the complete tensors (values of the velocity field for any discrete combination of radial and angular position) of the inflow wake and of the resulting angle of attack have been considered in present work, resulting in the feature sets FS2 and FS3. The wake and the angle of attack are provided for 25 radial positions and 60 angular positions.

The other additional features, which are necessary for a detailed characterization of the hydrodynamic/pressure field on the propeller blades, require the use of some numerical tools since their measurement is, generally, not feasible during MST. According to the aims of present work, the selected method should be able to compute the unsteady pressure distribution on the whole 3-D geometry of the propeller functioning in a non-uniform inflow field, since cavitation and radiated noise are strictly related to the pressure distribution and to its variation in time, at a limited computational cost necessary for design purposes.

For these reasons, an in-house developed Boundary Element Method has been used. BEM assumes incompressible, inviscid and irrotational fluid. These are the hypotheses which turn continuity and momentum equations, governing the flow dynamics, respectively into a Laplace equation for the velocity potential $\phi$ and into the Bernoulli theorem. A suitable choice of the boundary conditions allows to solve for steady and unsteady functioning, including sheet cavitation on both blade pressure and suction side, providing reliable estimations of the pressure distribution over the blades and of the induced flow field in proximity of the propeller.

The reliability and the effectiveness of Boundary Element Methods in predicting the steady and unsteady flow around marine propellers, also considering sheet cavitation, has been confirmed by several applications and benchmarks with overall satisfactory results. The pioneering applications of BEM for the characterization of propellers performances were those by [33 36] which progressively extended BEM capabilities to unsteady and cavitating flows also in the case of unconventional propellers like ducted propulsors. Midchord cavitation detachment, approximated tip vortex cavitation and the possibility to deal with supercavitating hydrofoils and propellers were included by [37, 38] and [39], while extensive validation against cavi- 
tation tunnel measurements were provided by [40, [41] and [42].

The current implementation of the method, which includes most of these features, has been validated accordingly. The reliability of propeller performances predictions for steady and unsteady functioning, also accounting for strongly non-homogeneous wake, was shown in [20, 43] and [21], demonstrating acceptable performance predictions even in very off-design functioning conditions [44]. Cavitating flows were considered in [45] for steady cases and in 22] for unsteady calculations, while supercavitating propellers were addressed in [46]. In addition, the Boundary Element Method of the University of Genoa, was successfully applied for the characterization of ducted [47] and tip loaded propellers [48] and in the framework of Simulation Based Design Optimization approaches [4, 5].

For the sake of completeness, Figure 5 summarizes the predicted propeller performances in homogeneous (steady) flow for one of the propellers considered in the present study. Calculations are compared with the measurements at the towing tank. The agreement, as expected from this kind of analyses, is satisfactory over the entire range of advance coefficient under investigation. Only torque, in correspondence of very loaded functioning conditions $(\mathrm{J} \approx 0.5-0.7)$ is slightly underestimated as the results of the approximations (no viscous forces, only empirical frictional corrections, no leading edge suction forces) accepted in the context of potential flow approaches).

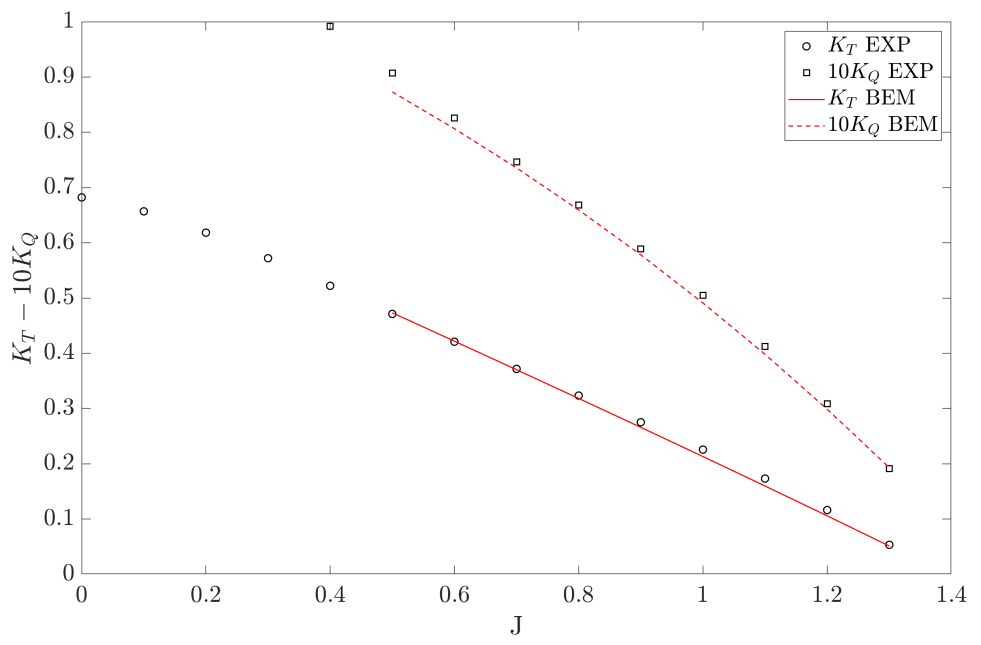

Figure 5: Predicted propeller performances (steady case) compared with towing tank measurements. 
In this work, the Boundary Element Method has been used to carry out unsteady calculations in order to take into account, in the definition of these additional features, the effect of the different non-homogeneous, 3D, wake fields considered in MSTs. In order to reduce the computational cost, indeed, numerical analyses with the BEM are limited to the case without cavitation, meaning that the development of the vapour cavity is not directly considered in the calculations and that the predicted propeller performance are not affected by the presence of the vapour cavity.

From the computations, in addition to the unsteady propeller performances, namely the thrust and the torque coefficients, the unsteady pressure coefficients $\left(C_{p}\right)$ on the blades (Eq. (7), where $p$ is the local pressure) and the unsteady blade circulation $(\mathcal{C})$ have been extracted. The distribution of the pressure coefficient is directly related to the occurrence of blade surface cavitation, while the circulation is related to the occurrence of vortex cavitation.

$$
C_{p}=\frac{p-p_{\text {static }, r e f}}{0.5 \rho(n D)^{2}}
$$

These quantities represent the feature sets FS4 and FS5, respectively. The pressure coefficients are given for 44 locations in chord-wise direction and 25 blade radial sections that represent the 1100 points per blade where the solution is compute. Sixty blade angular positions, corresponding to an equivalent time step of $6^{\circ}$ were instead considered to solve the unsteady functioning of the propeller in the non-uniform wakes of Figure 3 . These data, then, are organised in a 3D tensor. This means that the dynamic pressure acting on the whole surface of the blades during their rotation in a specific wake field is fully characterised. An example of the pressure distribution acting on the suction side and pressure side of the blades for a single propeller position is reported in Figure 6. In this example it is possible to observe some typical characteristics of these pressure distributions: the red area on the pressure side near the leading edge corresponds to the stagnation pressure while the blue area on the suction side at similar position is the typical negative pressure peak associated with the functioning of a hydrofoil with a positive angle of attack. As it can be seen, the pressure distributions on the different blades are not identical, and this is because the non-uniform propeller inflow is considered in the computation, hence each blade is functioning in a locally different flow field. The phenomenon of cavitation inception and development is very complex and involves also aspects that cannot be modelled reliably by the BEM. Nevertheless, it is already a good approximation to assume that cavitation occurs where the opposite of the local pressure coefficient is higher than the cavitation in- 


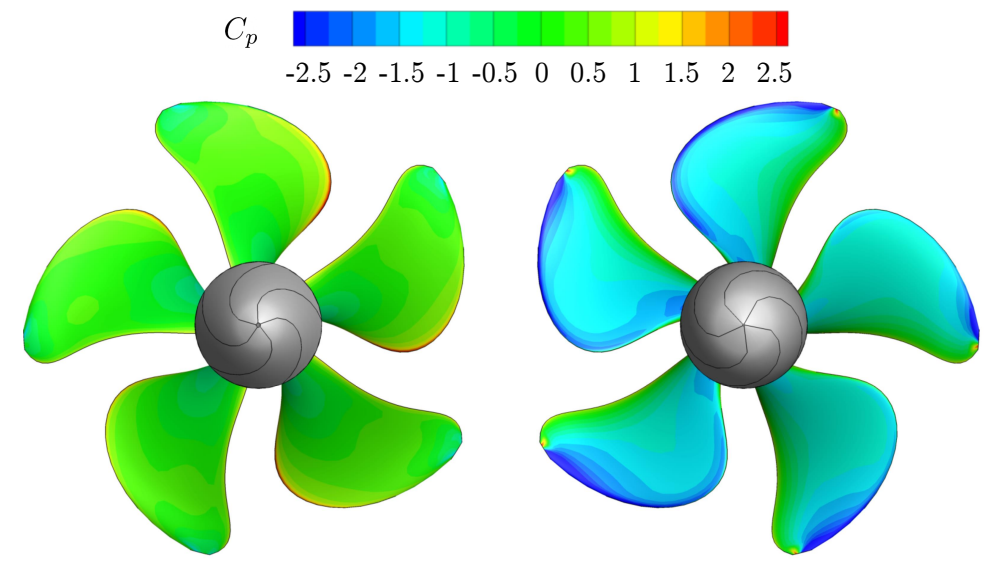

Figure 6: Predicted pressure distribution by BEM, propeller face (left) and back (right).

dex at that given functioning condition, meaning that the local pressure is lower than the vapour pressure. This is a cavitation inception estimation successfully applied for the design by optimization of conventional and unconventional propellers [5, 49]. Applying this criterion, it is possible to estimate the occurrence of cavitation on the blades, its location and to guess its extent. These considerations allow appreciating the valuable information that is provided to the model by this specific feature. Furthermore, it must be noticed that the pressure distribution on the blades is directly related to the geometry of the propeller, therefore this quantity implicitly takes into account also this aspect.

The circulation produced by a foil immersed in a fluid flow is directly related to the forces acting on the foil, and in particular to the lift, according to the well known Kutta-Joukowski theorem. Similarly, the circulation distribution on propeller blades correlates with the load acting on the blades and its distribution, which in turn is strictly related to the strength of the shed vortexes. Actually, the blade circulation at outer radii is the measure of the vortex strength also used in the PMs considered in present work to predict the tip vortex noise, as explained more in details in Section 3.2 .

The circulation is given for 25 blade radial sections, and the same 60 blade angular positions also considered for the pressure coefficients, resulting in a $2 \mathrm{D}$ tensor.

\subsection{Target definition}

The target of the models proposed in this work may be represented by a suitable description of propeller noise. In this view, frequency spectra of 
noise are a straightforward choice, being the most used representation of noise in many engineering fields.

Accordingly, two alternative target definitions are represented by the SL and RNL spectra, as defined in Section 2.1. These targets are named NSP4 and NSP5, respectively. The OTO band representation is used, resulting in two vectors of 24 elements corresponding to the noise levels measured for frequency bands in the range $0.4 \mathrm{kHz}$ to $80 \mathrm{kHz}$.

According to the theory of cavitation noise, correlations exist between the physics of cavitation and the main characteristics of the noise spectrum, such as the characteristic frequencies, the peak level, the high frequency levels and others. On the contrary, the behaviour of a single spectral row is more challenging to interpret. Consequently, to efficiently model the undergoing physical laws, it is necessary to analyse the values of the 24 elements of the proposed targets as a whole, including possible mutual interactions. This is achieved in present work by the method described in Section 3.3.

Besides, a further approach is considered, similar to that presented in [19]. According to this approach, only the main characteristics of the spectrum are considered as a further alternative definition of the target. This has a twofold objective: the first is to analyse the performance of the method with some alternative problem definitions, secondarily the physical models considered in present work address only some of these spectra characteristics instead of providing an estimate of the whole spectrum, hence to develop HMs, the same targets as the PMs are used.

The main characteristics of the noise spectrum are defined based on a simplified description of the spectrum, keeping only important information from the physical and practical point of view. The typical noise spectral shape for a propeller affected by suction side sheets and tip vortices cavitation is represented in Figure 7. It consist of a hump at low-mid frequencies and of an almost constant power decay moving towards higher frequencies. The maximum point in the spectrum and the hump, if present, is generally associated to the major part of the evolution of large cavities, such as growth and initial stage of collapse or cavity pulsation. In particular, the hump is present when vortex cavitation occurs and its associated to the pulsation of vortexes. The high frequency part of the spectrum is instead associated to the final stage of collapse and to the dynamics of smaller cavities. The decay law of the noise spectrum at these frequencies depends upon the dynamic of the sheet cavitation, in the current dataset, and only partially to vortex cavitation. The simplified spectrum is defined by the knowledge of only four points and the relevant levels and frequencies, as visible in Figure 7 . These points are detected automatically with a algorithm searching for the 
best fitting piece-wise function. However, the automatic identification of the center peak is rather challenging, because of the irregular shape of noise spectra, therefore results for the centre peak have been checked and adjusted manually. These frequencies and levels represent a further target definition, referred to as NSP2. As mentioned, this description of the noise spectrum

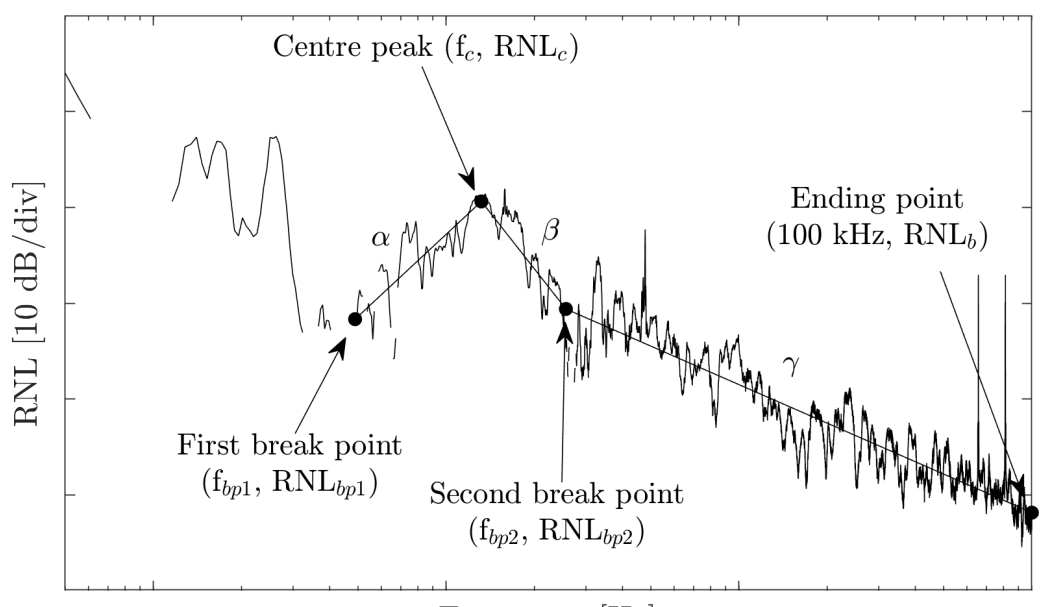

Frequency $[\mathrm{Hz}]$

Figure 7: Adopted spectrum simplification.

aims to focus on those spectral characteristics which better correlate with the physics of cavitation and the output of the physical models. This perfectly holds for the frequency and amplitude of the maximum peak in the spectrum, as well as for the level at the highest considered frequency that satisfactorily represents the power content of the high frequency noise. On the other hand, it is more difficult to correlate the behaviour of the other points with the physics of cavitation, and this could partially explain some of the difficulties observed in modelling these specific targets, as shown in [19].

To overcome this issue, an alternative description of the same simplified spectrum is defined based on the slopes of the broken-stick function; actually, their value is deemed to be more representative of the physics of cavitation, even if also their behaviour is not easy to interpret. By using these quantities, the simplified spectrum is identified by $f_{c}, \mathrm{RNL}_{c}, \mathrm{RNL}_{b}$ and the three slopes $\alpha, \beta, \gamma$. This last alternative targets representation is named NSP3. Lastly, NSP1, which includes only the centre peak $\left(f_{c}, \mathrm{RNL}_{c}\right)$ taken straight from NSP2, allows to focus the attention on the most crucial 
target and to compare the HMs with the PMs that predict this spectral characteristic.

\section{Modelisation}

In the proposed context, namely the estimation of the variables of the different noise spectra parametrizations based on a series of input variables characterising the propeller (see Table 4), a general modelisation framework can be defined, characterised by an input space $\mathcal{X}$, an output space $\mathcal{Y}$, and an unknown relation $\mu: \mathcal{X} \rightarrow \mathcal{Y}$ to be learned. In the specific case, $\mathcal{X}$ is composed by the FS reported in Table 4. In particular, the FS is composed by a series of scalar (FS1), a series of two-dimensional tensors (FS2, FS3, and FS5) and a three-dimensional tensor (FS4). On the other hand, the output space $\mathcal{Y}$, depends on the chosen parametrization (NSP1, NSP2, NSP3, NSP4, and NSP5). In this context, the authors define as model $h: \mathcal{X} \rightarrow \mathcal{Y}$ an artificial simplification of $\mu$. The model $h$ can be obtained with different kinds of techniques, for example requiring some physical knowledge of the problem, as in PMs, or the acquisition of large amount of data, as in DDMs, or both of them, as in HMs.

\subsection{Performance Measures}

Independently of the adopted technique, any model $h$ requires some data to be tuned (or learned) on the problem specificity and to be validated (or tested) on a real-world scenario. For these purposes, two separate sets of data $\mathcal{D}_{n}=\left\{\left(X_{1}, Y_{1}\right), \cdots,\left(X_{n}, Y_{n}\right)\right\}$ and $\mathcal{T}_{m}=\left\{\left(X_{1}^{t}, Y_{1}^{t}\right), \cdots,\left(X_{m}^{t}, T_{m}^{t}\right)\right\}$, where $X \in \mathcal{X}$ and $Y \in \mathcal{Y}$, need to be exploited, to respectively tune $h$ and evaluate its performances. It is important to note that $\mathcal{T}_{m}$ is needed since the error that $h$ would commit over $\mathcal{D}_{n}$ would be too optimistically biased since $\mathcal{D}_{n}$ has been used to tune $h$.

Note that, in this specific case, $Y \in \mathcal{Y}$ is not a simple scalar but it is a more complex structure since it is a characterisation of the noise spectra.

Let us suppose, for a moment, that $\mathcal{Y} \subseteq \mathbb{R}$, namely $Y \in \mathcal{Y}$ is a scalar.

In this case, the error that $h$ commits on $\mathcal{T}_{m}$ in approximating the real process is usually measured with reference to different indexes of performance [19]

\footnotetext{
${ }^{2}$ See Section 3.2

${ }^{3}$ The variable has been used to compute a feature for a PM

${ }^{4}$ In this case just the last elements of the vectors can be estimated with the PMs, see Section 3.2
} 


\begin{tabular}{|c|c|c|c|c|}
\hline $\begin{array}{l}\overrightarrow{0} \\
\text { है } \\
\text { ह }\end{array}$ & 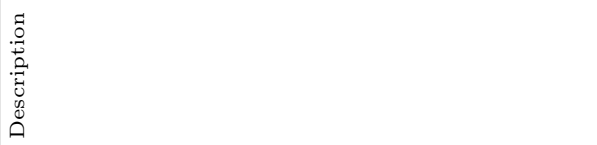 & 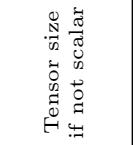 & 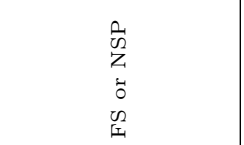 & 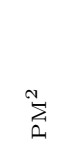 \\
\hline $\begin{array}{l}\mathrm{P} / \mathrm{D} \\
\mathrm{D} \\
\mathrm{BAR} \\
\mathrm{Z} \\
\mathrm{c} / \mathrm{D} \\
t_{\max / \mathrm{c}} \\
f_{\max / \mathrm{c}}\end{array}$ & \begin{tabular}{|l} 
Pitch ratio \\
Diameter \\
Blade area ratio \\
Number of blades \\
Chord ratio at $0.7 \mathrm{R}$ \\
Blade maximum thickness at $0.7 \mathrm{R}$ \\
Blade maximum camber at $0.7 \mathrm{R}$ \\
\end{tabular} & & $\begin{array}{l}\text { Geometry } \\
\text { (FS1) }\end{array}$ & $\begin{array}{l}\text { FS0 } \\
\text { FSo } \\
\text { FSO }\end{array}$ \\
\hline $\begin{array}{l}\alpha_{s} \\
\mathrm{~J} \\
\mathrm{~K}_{\mathrm{T}} \\
10 \mathrm{~K}_{\mathrm{Q}} \\
\mathrm{V}_{\mathrm{a}} \\
\mathrm{n} \\
\sigma_{v} \\
\sigma_{n} \\
\sigma_{\mathrm{tip}} \\
\end{array}$ & $\begin{array}{l}\text { Shaft angle } \\
\text { Advance coefficient } \\
\text { Thrust coefficient } \\
\text { Torque coefficient } \\
\text { Advance velocity } \\
\text { Propeller rotation } \\
\text { Cavitation index ref. to advance velocity } \\
\text { Cavitation index ref. to rotational speed } \\
\text { Cavitation index ref. to resultant speed at blade tip }\end{array}$ & & $\begin{array}{l}\text { Loading Parameters } \\
\text { (FS1) }\end{array}$ & $\begin{array}{l}\text { FS0 } \\
\text { FS0 } \\
\text { FSO }\end{array}$ \\
\hline $\begin{array}{l}\overline{\mathrm{w}} \\
\max \mathrm{w}_{07} \\
\mathrm{Wwd}_{07} \\
\left.D_{\theta} W\right|_{07} ^{-} \\
\left.D_{\theta} W\right|_{07} ^{+} \\
\max \mathrm{w}_{09}^{+} \\
\mathrm{Wwd}_{09} \\
\left.D_{\theta} W\right|_{09} ^{-} \\
\left.D_{\theta} W\right|_{09} ^{+}\end{array}$ & $\begin{array}{l}\text { Average } \mathrm{w} \\
\text { Wake maximum at } 0.7 \mathrm{R} \\
\text { Wake width at } 0.7 \mathrm{R} \\
\text { Left wake gradient at } 0.7 \mathrm{R} \\
\text { Right wake gradient at } 0.7 \mathrm{R} \\
\text { Wake maximum at } 0.9 \mathrm{R} \\
\text { Wake width at } 0.9 \mathrm{R} \\
\text { Left wake gradient at } 0.9 \mathrm{R} \\
\text { Right wake gradient at } 0.9 \mathrm{R} \\
\end{array}$ & & $\begin{array}{c}\text { Wake Parameter } \\
\text { (FS1) }\end{array}$ & \\
\hline $\begin{array}{l}\bar{\alpha}_{G 07} \\
\min \alpha_{G 07} \\
\max \alpha_{G 07} \\
\left.\theta\right|_{\max } \alpha_{G 07} \\
\bar{\alpha}_{G 09} \\
\min \alpha_{G 09} \\
\max \alpha_{G 09} \\
\left.\theta\right|_{\max \alpha_{G 09}} \\
\end{array}$ & $\begin{array}{l}\text { Circumferential average } \alpha_{G} \text { at } 0.7 \mathrm{R} \\
\text { Minimum } \alpha_{G} \text { at } 0.7 \mathrm{R} \\
\text { Maximum } \alpha_{G} \text { at } 0.7 \mathrm{R} \\
\text { Angular position of maximum } \alpha_{G} \text { at } 0.7 \mathrm{R} \\
\text { Circumferential average } \alpha_{G} \text { at } 0.9 \mathrm{R} \\
\text { Minimum } \alpha_{G} \text { at } 0.9 \mathrm{R} \\
\text { Maximum } \alpha_{G} \text { at } 0.9 \mathrm{R} \\
\text { Angular position of maximum } \alpha_{G} \text { at } 0.9 \mathrm{R} \\
\end{array}$ & & $\begin{array}{l}\text { Angle of Attack } \\
\qquad(\mathrm{FS} 1)\end{array}$ & \\
\hline $\mathrm{w}$ & Axial wake & $360 \times 31$ & $\begin{array}{c}\text { Wake Parameter } \\
\text { (FS2) }\end{array}$ & \\
\hline$\alpha_{G}$ & Geometric angle of attack & $360 \times 31$ & $\begin{array}{l}\text { Angle of Attack } \\
\text { (FS3) }\end{array}$ & \\
\hline $\mathrm{C}_{\mathrm{P}}$ & Coefficient of pressure on blade & $44 \times 25 \times 60$ & $\begin{array}{l}\text { BEM } \\
(\mathrm{FS} 4)\end{array}$ & $\mathrm{FSt}^{3}$ \\
\hline $\mathcal{C}$ & Blade circulation & $60 \times 25$ & $\begin{array}{l}\text { BEM } \\
\text { (FS5) }\end{array}$ & $\mathrm{FS} 3$ \\
\hline $\begin{array}{l}f_{\mathrm{c}} \\
\mathrm{RNL}_{\mathrm{c}} \\
f_{\mathrm{bp} 1} \\
f_{\mathrm{bp} 2} \\
\mathrm{RNL}_{\mathrm{bp} 1} \\
\mathrm{RNL}_{\mathrm{bp} 2} \\
\mathrm{RNL}_{\mathrm{b}} \\
\alpha \\
\beta \\
\gamma \\
\mathrm{SL}_{1 / 3} \\
\mathrm{RNL}_{1 / 3}\end{array}$ & $\begin{array}{l}\text { Central peak frequency } \\
\text { Noise level at } f_{\mathrm{c}} \\
\text { Frequency of the first breakpoint } \\
\text { Frequency of the second breakpoint } \\
\text { Noise level at } f_{\mathrm{bp} 1} \\
\text { Noise level at } f_{\mathrm{bp} 2} \\
\text { Noise level at ending frequency }(100 \mathrm{kHz}) \\
\text { Slope between breakpoint } 1 \text { and central peak } \\
\text { Slope between central peak and breakpoint } 2 \\
\text { Slope between breakpoint } 2 \text { and last point } \\
\text { Source Levels in one-third octave bands } \\
\text { Radiated Noise Levels in one-third octave bands }\end{array}$ & $\begin{array}{l}24 \\
24\end{array}$ & $\begin{array}{l}\text { NSP1, NSP2, NSP3 } \\
\text { NSP1, NSP2, NSP3 } \\
\text { NSP2 } \\
\text { NSP2 } \\
\text { NSP2 } \\
\text { NSP2 } \\
\text { NSP2, NSP3 } \\
\text { NSP3 } \\
\text { NSP3 } \\
\text { NSP3 } \\
\text { NSP4 } \\
\text { NSP5 }\end{array}$ & $\sqrt{4}$ \\
\hline
\end{tabular}

Table 4: Dataset variables description. 
- the Mean Absolute Error (MAE) is computed by taking the absolute loss value of $h$ over $\mathcal{T}_{m}$

$$
\operatorname{MAE}(h)=\frac{1}{m} \sum_{i=1}^{m}\left|h\left(X_{i}^{t}\right)-Y_{i}^{t}\right|
$$

- the Mean Absolute Percentage Error (MAPE) is computed by taking the absolute loss value of $h$ over $\mathcal{T}_{m}$ in percentage

$$
\operatorname{MAPE}(h)=\frac{100}{m} \sum_{i=1}^{m}\left|\frac{h\left(X_{i}^{t}\right)-Y_{i}^{t}}{Y_{i}^{t}}\right| ;
$$

- the Pearson Product-Moment Correlation Coefficient (PPMCC) measures the linear dependency between $h\left(X_{i}^{t}\right)$ and $Y_{i}^{t}$ with $i \in\{1, \cdots, m\}$

$$
\operatorname{PPMCC}(h)=\frac{\sum_{i=1}^{m}\left(Y_{i}^{t}-\bar{Y}\right)\left(h\left(X_{i}^{t}\right)-\hat{\bar{Y}}\right)}{\sqrt{\sum_{i=1}^{m}\left(Y_{i}^{t}-\bar{Y}\right)^{2}} \sqrt{\sum_{i=1}^{m}\left(h\left(X_{i}^{t}\right)-\hat{\bar{Y}}\right)^{2}}},
$$

where $\bar{Y}=\frac{1}{m} \sum_{i=1}^{m} Y_{i}^{t}$ and $\hat{\bar{Y}}=\frac{1}{m} \sum_{i=1}^{m} h\left(X_{i}^{t}\right)$.

Other state-of-the-art measures of error exist (such as R-squared and or the Mean Square Error) but, from a physical point of view, they give a complete description of the quality of the model and adding more measures would make the results less readable.

Unfortunately, in the proposed application, $\mathcal{Y} \subseteq \mathbb{R}^{p}$ is a vector representing a parametrization of the cavitation noise spectra. For this reason, to provide a value which reasonably represents the error that $h$ commits on $\mathcal{T}_{m}$ when $Y \in \mathcal{Y}$ is a parametrization of the cavitation noise spectra, it is necessary to use a more suitable error measure which targets one of the particular parametrizations (NSP1, NSP2, NSP3, NSP4, and NSP5).

For what concerns the NSP4 and NSP5 the authors will redefine the MAPE as the average MAPE among the different parameters which compose the parametrizations. This can be done since NSP4, and NSP5 count homogeneous quantities (see Section 2) and the average MAPE well describes the quality of $h$, since it represents the average difference between actual and predicted spectra.

For what concerns NSP2 and NSP3 the approach adopted for NSP4 and NSP 5 cannot be directly applied but the error can be measured again as an average difference between spectra. Actually, keeping in mind that the parameters of NSP2 and NSP3 allow defining a spline which simplifies 
the noise spectrum (see Section 2), it is possible to redefine the MAPE for NSP2 and NSP3, as the average percentage distance between the actual (derived from $Y$ ) and predicted (derived from $h(X)$ ) splines, which in turn approximate the actual and predicted spectra. Finally, NSP1 represents just a point of the spectrum, therefore it is not possible to define the MAPE based on the distance between actual and predicted spectra for this target. In this case, the MAPE is defined as the average of the MAPE computed for the two parameters composing NSP1, namely the frequency and the level of the spectral hump. Due to this, the error defined for NSP1 does not compare rigorously with the error estimated for all the other targets.

\subsection{Physical Models (PMs)}

PMs are derived from the physical theories and relevant equations describing the phenomenon of interest, under certain assumptions making the solution of the equations affordable without the need for sophisticated computational tools. Although the structure of these formulations is derived from physical equations, experimental data are used to tune the formulations to deal with real world problems. For this reason, these methods are usually referred to also as semi-empirical methods. Two main physical models are considered in present work: the first one models the noise generated by the resonance of a cavitating tip vortex, the second regards the noise due to sheet cavitation.

Cavitating vortex noise. The resonance of the tip vortex cavity is assumed to be the main responsible for the low frequency hump typically observed in propeller noise spectra, when vortex cavitation is present, as depicted in Figure 7. Therefore, this model is used to predict the central frequency $f_{\mathrm{c}}$ and noise level $\mathrm{RNL}_{\mathrm{c}}$ of the centre peak. Two sub-models compose the vortex noise model:

I The model representing the relation between the dimension of the vortex (namely the cavitating radius $r_{c}$ ) and its characteristic noise.

II The vortex model describing the vortex velocity given a measure of the vortex strength.

The formulation adopted to describe the relationship between the pulsation frequency of a cavitating vortex and its radius can be derived from the analysis of the pulsation of a single bubble of gas immersed in an infinite fluid domain. The relation takes the form of Eq. (11)

$$
\frac{f_{\mathrm{c}}}{n Z}=c_{1} \frac{1}{r_{c} / D} \frac{\sqrt{\sigma_{t i p}}}{Z}+c_{2}
$$


where $r_{c}$ is the cavity radius, $Z$ is the number of blades, $\sigma_{t i p}$ is the cavitation number evaluated at the blade tip, $c_{1}$ and $c_{2}$ are unknown constants whose values can be determined by fitting on experimental data, as done in [50].

The amplitude of the noise generated by vortex pulsation depends again on the cavity radius. The formulation used in present work has been derived following an approach similar to that proposed by [7]. The original formulation gives the noise level in $\mathrm{dB}$ as a function of some parameters describing the propeller and its functioning condition. In the formulation used in present work, the noise level in $\mathrm{dB}$ is given as a function of the vortex cavity radius, the propeller diameter and the number of blades, as shown in Eq. 12

$$
\mathrm{RNL}_{\mathrm{c}}=a_{p}+20 \log _{10}\left[\left(\frac{r_{c}}{D}\right)^{k} \sqrt{Z}\right],
$$

where $a_{p}$ and $k$ are again unknown constants to be found through data fitting.

Both formulations used to compute the frequency and the noise amplitude of vortex pulsation require the knowledge of the cavity radius. This radius can be found as the radial distance $r$ from the vortex axis, where the local pressure equals the water vapour pressure; the local pressure can be determined if the velocity field around the vortex is known and this requires the use of a vortex model.

Within the several vortex models available in the literature, the one described in [51] has been used to estimate the radial distribution of the azimuthal velocity component. This model is based on the assumptions of 2-D and axisymmetric flow, implying constant axial velocity, negligible radial velocity with respect to the tangential velocity, and constant velocity distribution in the azimuthal direction. According to this model, the azimuthal velocity is given by:

$$
v(r)=\left\{\begin{aligned}
1.0939 \frac{\mathcal{C}_{\infty}}{2 \pi r}\left\{1-\exp \left[-\beta\left(\frac{1.4 r_{\nu}}{D / 2}\right)^{p}\right]\right\} \times & \\
\times\left\{1-\exp \left[-\zeta\left(\frac{r}{r_{\nu}}\right)^{2}\right]\right\} & r \leq 1.4 r_{\nu} \\
\frac{\mathcal{C}_{\infty}}{2 \pi r}\left\{1-\exp \left[-\beta\left(\frac{r}{D / 2}\right)^{p}\right]\right\} & r>1.4 r_{\nu}
\end{aligned}\right.
$$

where $\mathcal{C}_{\infty}$ is the vortex strength, $\zeta$ is a prescribed parameter taking the value of 1.2564 , and $p$ has been fixed at 0.75 as suggested by [51]. The coefficient $\beta$ has to be found by least square fitting on data. 
The viscous core radius $r_{\nu}$ is an input and may differ for every propeller and functioning condition. Since direct measurements are not available for present cases, literature values reported in [52] have been exploited. These values have been scaled according to the procedure proposed by [53] to take into account the different Reynolds number.

This scaling provides a first reasonable estimate of the viscous core for the current test cases, however, as also suggested in [54, it could be useful to adjust the obtained values by means of a constant multiplication factor, to better fit the formula to the data. The factor used in present work is equal to 0.5 .

Eventually, the vortex strength $\mathcal{C}_{\infty}$ is needed to compute the vortex velocity distribution using Eq. (13). In this work, this quantity has been computed by BEM in correspondence to the blade section at $r / R=0.95$. The circulation, for a propeller operating in a non-uniform inflow, depends on the blade angular position. The average value on the whole revolution has been used, multiplied by a factor 0.7 used again to fit the data.

At this point, the azimuthal velocity distribution in the vortex radial direction is known. Under the previously mentioned hypotheses, the pressure distribution can be predicted integrating the momentum equation in the radial direction [55], therefore the cavitating radius can be found.

In summary, the structure of the whole physical model for the tip vortex noise is represented by the flow chart in Figure 8; the vortex model, represented by Eq. (13), is used to obtain the cavitating radius based on some input data, successively Eq. (11) and Eq. (12) are used to derive the noise based on the knowledge of the cavitating radius.

The different equations included in the model involves the intensive use of data fitting to determine the value of different parameters that are not known a priori. In principle, the vortex flow model should be tuned on flow quantities (e.g. velocity distributions and the cavitating radii), while the relations between the cavitating radius and the resulting noise should be tuned on noise data. Since the vortex flow quantities were not measured in present campaigns, all the formulations have been tuned on the experimentally measured $f_{\mathrm{c}}$.

High frequency noise. The second model is used to predict the contribution of sheet cavitation that is assumed to dominate measured noise spectra in the frequency range $10 \mathrm{kHz}$ to $80 \mathrm{kHz}$. The model chosen is Brown's empirical 


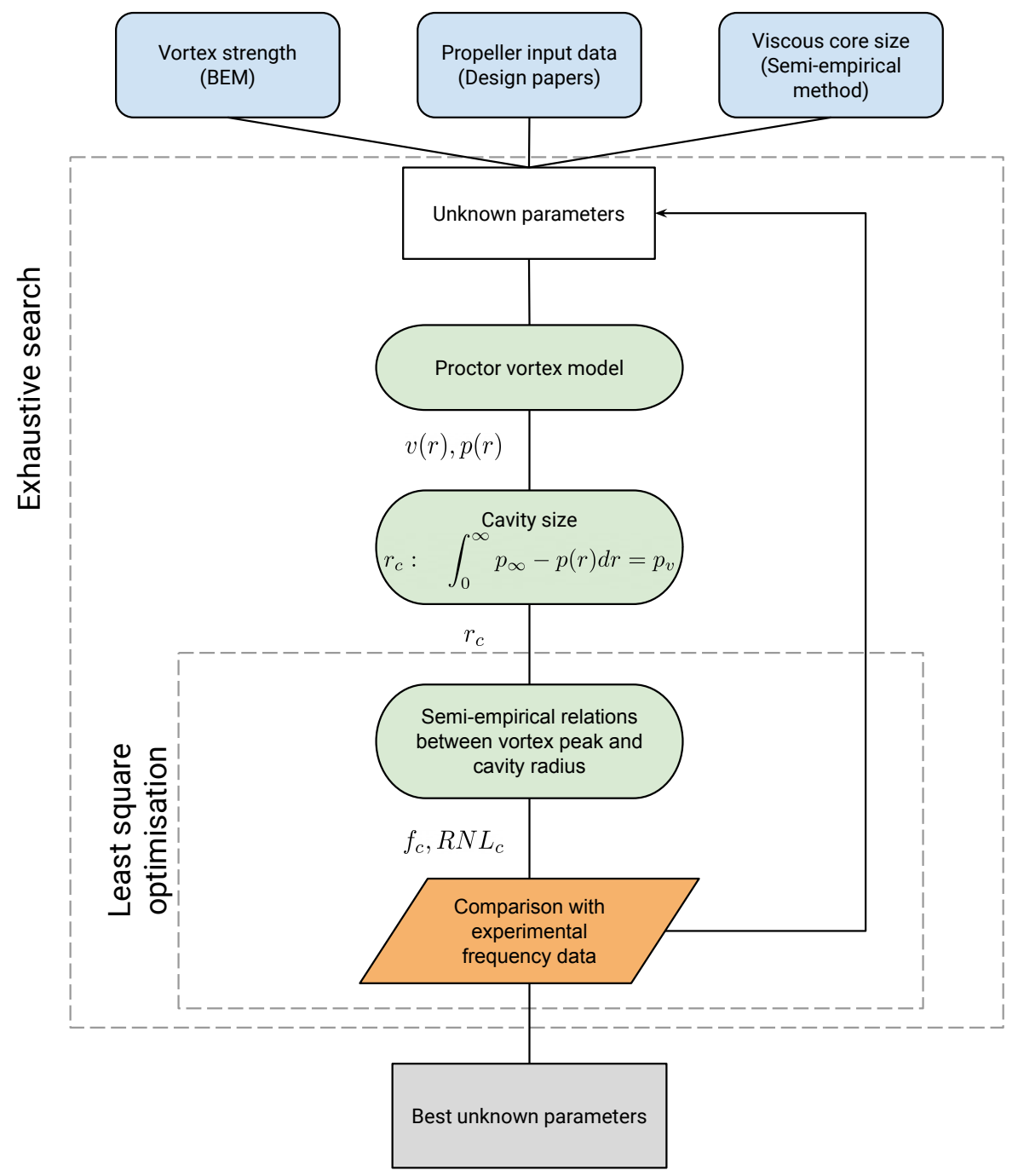

Figure 8: Schema of the tuning algorithm for the prediction of the vortex peak. 
formula discussed in [9].

$$
L=K+\eta\left[10 \log _{10}\left(Z D^{4} n^{3} f^{-1}\right)+10 \log _{10}\left(\frac{A_{C}}{A_{D}}\right)\right],
$$

where $A_{D}$ is the propeller disk area, $f$ is the frequency and $L$ is the noise $\mathrm{dB}$ level. The cavitating area $A_{C}$ has been derived from the distribution on the blades of the pressure coefficient $C_{p}$, assuming that cavitation is present where $C_{p}<-\sigma_{n}$. This approximation neglects bubble dynamics and the real development of cavitation, therefore the predicted area is expected to be underestimated.

In the formula, the unknown parameters are the constants $K$ and $\eta$ which have been found by fitting to the experimental radiated noise levels and source levels. It is not surprising to find that $K$ is the same value both for the RNL and the SL; at higher frequencies, the effect of the confined environment is practically negligible.

\subsection{Data Driven Models (DDMs)}

In this section the authors will present the proposed DDMs for predicting the different NSPs based on the different FSs (see Table 4) exploiting the data presented in Section 2 .

Even if the scenario of this paper is slightly different with respect to the one of [19] (see Section 1), a first idea could be to exploit the same methodology for defining new DDMs and HMs and adapt it to the scope of this work. Unfortunately, for the reasons that will be clarified in this section [23, 56], this approach would result in very low performance, in terms of accuracy, as will be shown in the experimental results of Section 4.2 . The reasons behind this decay in performance need to be searched in the philosophy behind the methodology proposed in [19] that, from now on, will be named as Conventional DDMs (CDDMs) or Shallow DDMs (SDDMs). CDDMs rely on the simple pipeline presented in Figure 9(a).

- from the available inputs, i.e. the propeller characteristics and operational conditions, the raw information about the FSs of Table 4 are extracted;

- from the raw FSs, experts of the problem together with data scientists extract a series of rich features, that should be able to provide all the information about the desired output, in this case the different parameters of the NSPs (this process is called Feature Engineering);

- a functional form of the predictive model, the SDDM, is defined by the data scientist. Then the parameters of the SDDMs are learned from 


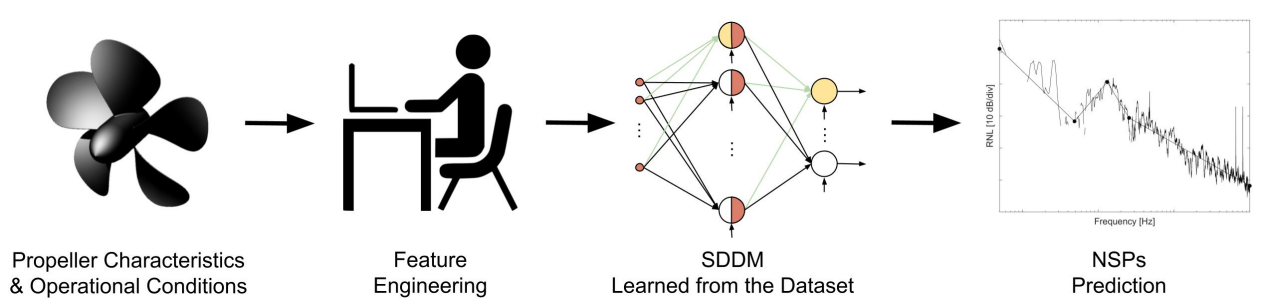

(a) CDDMs or SDDMs.

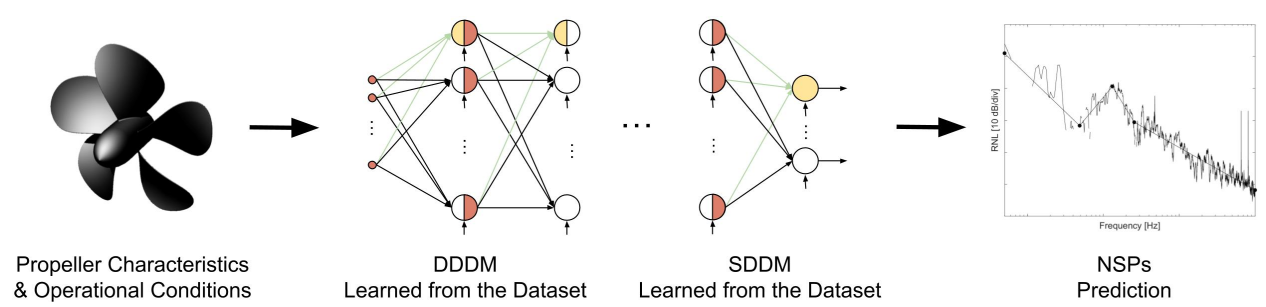

(b) ADDMs or DDDMs.

Figure 9: Conventional (Shallow) DDMs vs Advanced (Deep) DDMs.

the input/output samples, called dataset, where the input is coded with the features engineered in the previous step;

- finally the learned model can be exploited to make prediction about the NSPs.

This approach is very effective under a simple, but quite strict, assumption: the feature engineered by the experts should be rich enough to describe the phenomena, but characterised by a cardinality not too high compared to the number of samples of the dataset [23, 56. If only FS1 is available, somehow analogously to the work of [19, then CDDMs would be the correct choice. As a matter of fact, in this case also FS2, FS3, FS4, and FS5 are available, and exploiting the CDDMs of [19] would result in an exploding number of features, because of the FSs intrinsic cardinality, and the model would be not able to learn the correct model with a dataset of limited cardinality like the one available for this study (see Section 2). Moreover, the extraction of rich and representative features from the FS2, FS3, FS4, and FS5 is a complex task not suited for a human expert.

For these reasons, in this paper, the authors will exploit an Advanced DDMs (ADDMs) or Deep DDMs (DDDMs). ADDMs rely on the pipeline presented in Figure 9(b)

- as for the CDDMs, from the available inputs, (propeller characteristics and operational conditions), the raw information about the FSs of 
Table 4 are extracted;

- contrary to the CDDMs, experts of the problem together with data scientists do not perform a Feature Engineering phase, but they define a functional form of the model, namely a structure of the model, to be learned from the data. This structure is composed of two levels: a first level (DDDMS) is dedicated to learning the features to be provided to the same SDDM exploited for the CDDMs;

- from the dataset, both the SDDM and the DDDM parameters are learned;

- finally the learned model can be exploited to make prediction about the NSPs.

The main differences between the CDDMs and the ADDMs rely on the fact that, in the ADDMs there is just minimal intervention of experts and data scientists in the definition of the model. In fact, in ADDMs, as it will be shown in this section, just the functional form of the features must be designed, while in CDDMs the features are basically handmade, everything else is learned from the dataset.

In this work, the CDDMs are not described in details, nevertheless, all the relevant details are reported in the original work of [19]. For completeness, the authors just recall that the CDDMs proposed in [19] is a combination of features engineered by experts [19] plus a Kernel Regularised Least Squares (KRLS) [57] model plus a Feature Reduction phase [58] plus an advance Model Selection phase [59].

Instead, from now on, a detailed description of the proposed ADDMs is reported, starting from the basic principles that guided the definition of the proposed functional form until the final proposed model. In particular, the authors will first explain the building blocks of the proposed ADDM, and then they will show how to combine them to derive the proposed architecture and solve the problem faced in this work. For simplicity, if not specified otherwise, the proposed ADDMs is simply referred as DDMs since, as it will be shown in Section 4.2, the ADDMs are the most effective ones for the purpose of this paper.

Let us start by making the hypothesis that $\mathcal{X} \subseteq \mathbb{R}^{q}$ with $q \in \mathbb{N}^{*}$, namely the input space is composed by different features stacked together in a vector (e.g. when only FS1 is considered), and that $\mu$ is a simple linear function. Note that, for each one of the different NSPs $\mathcal{Y} \subseteq \mathbb{R}^{p}$ with $p \in \mathbb{N}^{*}$, namely the output space is composed by different features stacked together in a vector. In this case, the best functional form of the model can be defined as 
follows

$$
h(X)=W X+B
$$

where $W \in \mathbb{R}^{p \times q}$ and $B \in \mathbb{R}^{p}$, namely the functional form of the model is a multiple linear model in the space defined by $\mathcal{X}$ and $\{W, B\}$ are the parameters of the model. The functional form of the model can also be interpreted as $p$ different neurons reacting to the input stimulus in different ways based on the different weights. The model of Eq. (15) is also graphically depicted in Figure 10. $\{W, B\}$ need to be tuned in such a way that $h$ is a

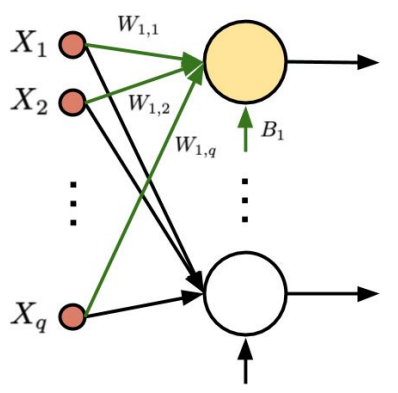

Figure 10: Multiple linear model functional form for $h$. In red are identified the inputs, in green the weights, and in yellow the output of one neuron of the architecture.

good representation of $\mu$. However, the quality of $h$ in representing $\mu$ is defined by the index of performance defined in Section 3.1 for each of the NSPs computed on $\mathcal{D}_{n}$. The authors will refer to these measure of accuracy with the symbol $\hat{\mathrm{L}}\left(h, \mathcal{D}_{n}\right)$, namely the Empirical Error $\hat{\mathrm{L}}$ of the model $h$ on the dataset $\mathcal{D}_{n}$. Consequently, the best parameters of $h$, namely $\mathrm{P}(h)$ (in the case of Eq. 15 $\mathrm{P}(h)=\{W, B\})$, are the ones which minimise the following problem

$$
h^{*}: \min _{\mathrm{P}(h)} \hat{\mathrm{L}}\left(h, \mathcal{D}_{n}\right) \text {. }
$$

In general, Problem (16) can be convex or not, depending on the functional forms of $h$ or $\hat{\mathrm{L}}\left(h, \mathcal{D}_{n}\right)$ [60, 61]. For this reason, if, at least, $\hat{\mathrm{L}}(h)$ is differentiable in $\mathrm{P}(h)$ (e.g. the model of Eq. (15) should be differentiable in $\{W, B\}$ ), it is possible to exploit one of the various forms of the gradient descend algorithm 23] (e.g. SGD, RMSprop, Adagrad, Adadelta, Adam, etc.) to find the best set of $\mathrm{P}(h)$. Note that each algorithm has hidden hyperparameters that need to be tuned to reach satisfying solutions [23] (e.g. Learning Rates, Momentum, Batch Sizes, etc.). Another critical aspect which deeply affects the 
results of the optimisation process in gradient-based methods is the starting point, or initialisation, of $\mathrm{P}(h)$; also in this case many options exist, but they are grouped in two big families [23]: the first one is the deterministic initialisation (e.g. Random Normal, Random Uniform, LeCun, Glorot, He, etc.) while the second one is the learned initialisation (e.g. Autoencoders, etc.).

This approach of Problem (16) is known as Empirical Risk Minimisation (ERM) 62]. However, ERM is usually avoided in DDMs as it leads to severe overfitting of the model on the training dataset. As a matter of fact, in this case the process of learning the $\mathrm{P}(h)$ could choose a model good just for describing $\mathcal{D}_{n}$ which has been exploited to learn $\mathrm{P}(h)$ (including noise, which afflicts $\mathcal{D}_{n}$ ). In other words, ERM implies memorisation of data rather than learning from them. For this reason, some regularisation effect in Problem (16) has been included, allowing to find a $\mathrm{P}(h)$ good enough to both learn from $\mathcal{D}_{n}$ and generalise, namely have good performance, also on $\mathcal{T}_{n}$. Many ways exist to introduce this regularisation [23] (e.g. Early Stopping, Weights Decay, Dropout, etc.) and with a little abuse of notation all of them will be modelled by modifying Problem (16) into the following one

$$
h^{*}: \min _{\mathrm{P}(h)} \hat{\mathrm{L}}\left(h, \mathcal{D}_{n}\right)+\lambda \mathrm{R}(h),
$$

where $\mathrm{R}(h)$ represents the regularisation term and $\lambda \in[0,+\infty)$ represents the regularisation hyperparameter, that need to be tuned, in order to find the best trade-off between ability of $\mathrm{P}(h)$ to learn from $\mathcal{D}_{n}$ and to generalise.

The limitations that are still considered are the hypothesis of the linearity of $\mu$ and then the linearity of functional form of the model presented in Eq. (15). In order to address this issue, it is possible to combine multiple linear models in this way

$$
h(X)=W_{2} \Gamma\left(W_{1} X+B_{1}\right)+B_{2},
$$

where $\Gamma^{5}$ is an activation function [23] (e.g. Sigmoid, Hyperbolic Tangent, Rectified Linear Unit, etc.), $W_{1} \in \mathbb{R}^{h \times q}$ with $h \in \mathbb{N}^{*}, B_{1} \in \mathbb{R}^{h}, W_{2} \in$ $\mathbb{R}^{p \times h}$, and $B_{2} \in \mathbb{R}^{p}$. The model of Eq. $(18)$ is also graphically depicted in Figure 11. If $\Gamma$ is, for example, a Sigmoid or a Hyperbolic Tangent

\footnotetext{
${ }^{5}$ With a little simplification the authors exploited the same activation function element of the vector $W_{1} X+B_{1}$ but in general it is possible to use different activation functions.
} 


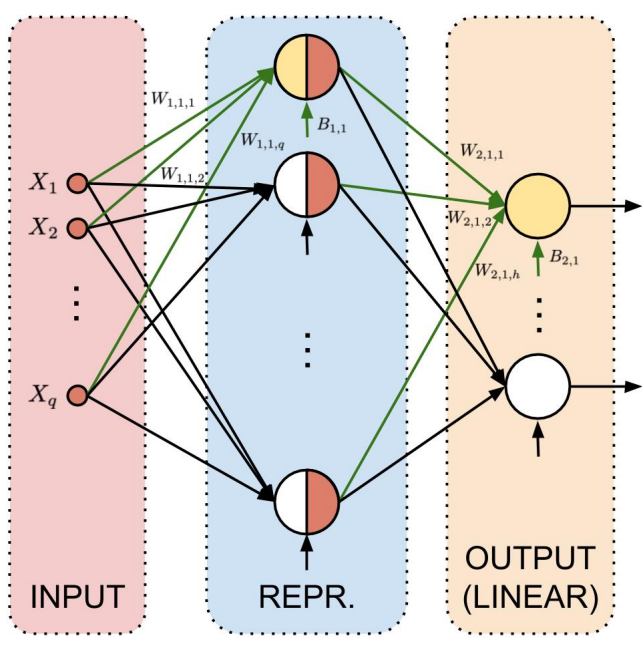

Figure 11: Shallow DDM.

it is possible to prove that, for $h$ large enough, the model Eq. 18 can describe every possible function mapping points from $\mathbb{R}^{q}$ to $\mathbb{R}^{p}[23$. The type of activation function and $h$ are hyperparameters which characterise the architecture that need to be tuned. The model of Eq. $(18)$ is also called Shallow (SDDM) since it can be interpreted as the concatenation of multiple neurons with a single hidden layer of neurons. Note that this SDDM is somehow analogous to the one exploited in the CDDMs where the hidden layer represents the feature mapping, which is deterministic in CDDMs, while in this case is learned from the data and the last layer is a simple linear function (note that, if the representation is good, linear functions are powerful enough). Since the structure of the brain is not so simple but it has a deeper structure, in the last years [23] Deep Models (DDDMs) have been developed and have shown to outperform many SDDMs in many real world problems involving natural signals [23] (e.g. Image and Video Recognition, Natural Language Processing, Speech Recognition, etc.). In this case, the model is a concatenation of multiple neurons in a series of multiple hidden layers as follows

$$
h(X)=W_{H} \Gamma_{H}\left(\cdots \Gamma_{2}\left(W_{2} \Gamma_{1}\left(W_{1} X+B_{1}\right)+B_{2}\right) \cdots\right)+B_{H},
$$

where $\Gamma_{2}^{6}$ with $i \in\{1, \cdots, H\}$ and $H \in \mathbb{N}^{*}$ are the different activation

\footnotetext{
${ }^{6}$ With a little simplification the authors exploited the same activation function in each hidden layer but in general each hidden layer may exploit different activation functions.
} 
functions at each layer, $W_{1} \in \mathbb{R}^{h_{1} \times q}, B_{1} \in \mathbb{R}^{h_{1}}, W_{H} \in \mathbb{R}^{p \times h_{H-1}}$ with $h_{H-1} \in$ $\mathbb{N}^{*}, B_{H} \in \mathbb{R}^{p}, W_{i} \in \mathbb{R}^{h_{i} \times h_{i-1}}$, and $B_{i} \in \mathbb{R}^{h_{i}}$ with $i \in\{2, \cdots, H-1\}$. The type of activation function, $H$, and $h_{i}$ with $i \in\{1, \cdots H-1\}$ are hyperparameters which characterise the architecture that need to be tuned. The model of Eq. (19) is also graphically depicted in Figure 12 . Note that

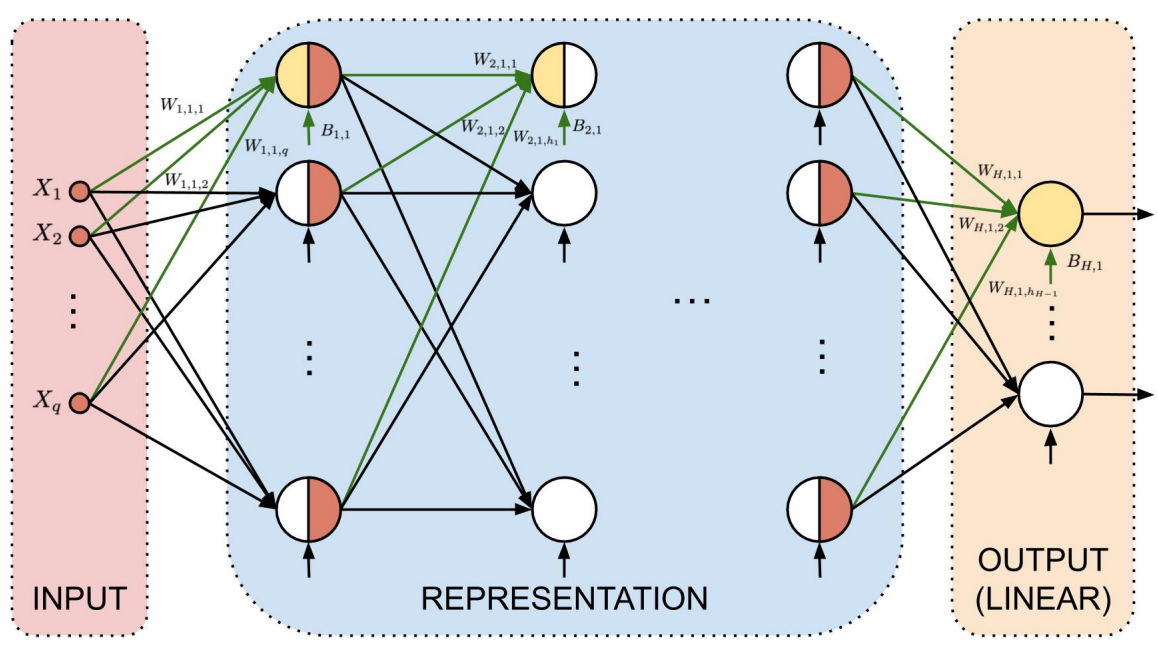

Figure 12: Deep DDM.

in DDDMs, the representation that can be learned from the data is richer with respect to the SDDMs since it allows building more complex and, in principle, richer structures [23].

The problem of the models of Eqns. (18) and (19) is that the cardinality of the matrices of parameters explodes too fast (curse of dimensionality) and then, in order to be able to learn them, millions of samples need to be available [23]. In this case, this is not possible since the cardinality of the dataset is very limited and fixed as described in Section 2 and this is also the reason why the CDDMs cannot be exploited.

One way to overcome this problem is to exploit the solution of the Extreme Learning Machines (ELMs) [63, 64] which show that the ability to learn a good model from data, in the form of Eqns. (18) and (19), improves dramatically, in the case of limited number of samples, when $W_{1}$ for model Eq. (18) and $W_{1}, \cdots, W_{H-1}$ for the one of Eq. (19) are simply set randomly. Counterintuitive as it may seem, this solution represents the state of the art in many real world applications [63, 64].

Another way to overcome the curse of dimensionality is to introduce the concept of sparsity on the architecture. Sparsity means that many weights in 
the architecture are zero or missing (an example, for simplicity on a SDDM, is reported in Figure 13).

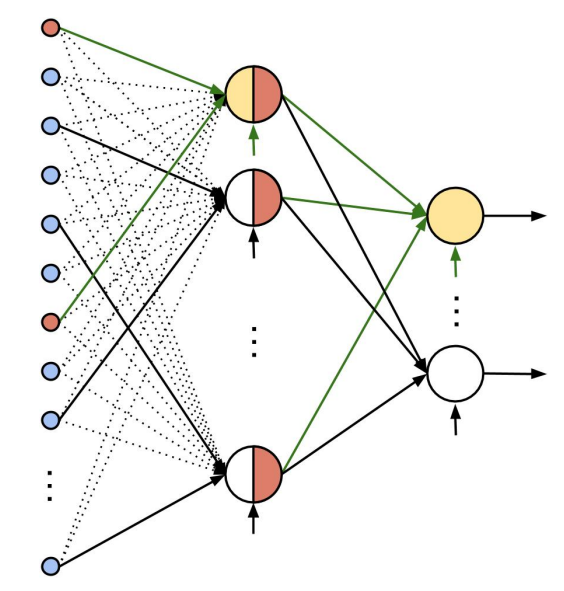

Figure 13: Sparse version of the SDDMs of Figure 11 Dotted rows means that that weight is set to zero, namely the connection is dropped.

This property can be achieved in two ways.

The first one, the naive one, is to use as $\mathrm{R}(h)$ the L1 [65] or the L1-L2 [66] regularisation which implies a sparse solutions in Problem (17). Unfortunately, this approach does not really mitigate the curse of dimensionality and presents some intrinsic limitations [23]. The reasons of these limitations lie in the fact that, simply adding a regularisation, does not help the model in reducing the intrinsic dimensionality of the space of the parameters since it still has to learn what parameters need to be set to zero.

The second option is to exploit the intrinsic structure of the FS. Until this point the hypothesis that was made considers that the input space is composed by different features stacked together in a vector (e.g. when just FS1 is considered) but, in the specific problem, some parts of the FS have a particular structure. In fact, FS2, FS3, and FS5 are two-dimensional (2D) tensors while FS4 is a three-dimensional (3D) tensor (please refer to Table 4 for simplicity or to Section 2 for the detailed explanation of these FSs). These 2D and 3D-tensors, contrarily to the FS1, have a particular property. Apart from the specific value of each particular element in the tensor, also the location in the tensor has a meaning related the tensor's construction (see Section 2). In a simpler case, like grayscale images, which can be represented as a $2 \mathrm{D}$-tensor, pixels which are close to each other have some proximity property, called structure, which can help, for example, in distinguish things that are close or distant from each other or pixel which 
belongs to the same object [23. In this case, it does not make any sense to stack neurons in layers which react to all the elements in the tensor contemporary (basically to build a structure like the ones in Figs. 10, 11, and 12 but each neuron should react to particular portion of the tensors, ignoring the other ones. This corresponds to deterministically set to zero some weights of the neurons and then, to fully process the tensor, to stack neurons which react to different portion of the tensors (namely a structure like the one of Figure 13 is deterministically defined). The problem that remains to be solved is how to define a structure of these neurons, and for this reason the authors will rely on a simple idea [23]: each neuron should react to part of the tensors which are close, in some sense, to each other. This idea comes from the use of convolution which Gabor applies in image processing [67, which emulates how the brain process the images to detect, for example, objects, distances, and contours. The only difference here is that these filters, instead of being deterministically defined to react to certain stimulus, are learned from the data. An example of the structure of the network for a 2D-convolution layer is reported in Figure 14 where, for simplicity, just two neurons are fully depicted. The architecture has

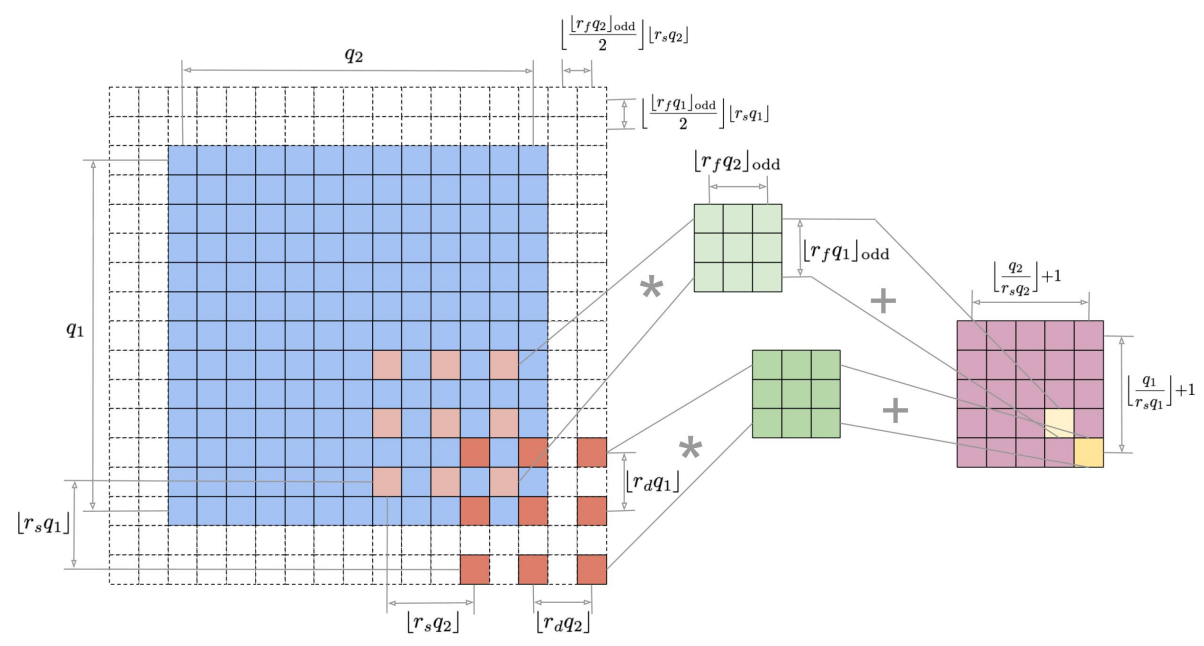

Figure 14: Convolution on a two-dimensional tensor. The tensor has been indicated in blue, the learned filter (the sparse weights) in green, the output of one sparse neuron in yellow, the resulting two-dimensional tensor in purple, the padding in white.

several parameters. The input space, being a 2D-tensor, is a matrix of size $\mathbb{R}^{q_{1} \times q_{2}}$. The patch, or the size of the filter to be learned, is $\mathbb{R}^{\left\lfloor r_{f} q_{1}\right\rfloor_{\text {odd }} \times\left\lfloor r_{f} q_{2}\right\rfloor_{\text {odd }}}$ where $r_{f} \in(0,1)$ is an hyperparameter which regulates the ratio between 
the size of the 2D-input tensor and the filter while $\lfloor\cdot\rfloor_{\text {odd }}$ represents the closer smaller odd number. The padding is the addition of elements at the border of the tensor to mitigate the edge effects, and its size is depicted in Figure 14 (in this case the zero padding has been used, but also other types of padding exists like the "mirror" or "same" padding). The stride is the movement step of the filter on the tensor which is $\left\lfloor r_{s} q_{1}\right\rfloor$ along the first dimension of the tensor and $\left\lfloor r_{s} q_{2}\right\rfloor$ along the second dimension, $r_{s} \in$ $(0,1)$ is and hyperparameter which regulates this movement. The dilation is a further sparsity capability of the filter reaction, which is $\left\lfloor r_{d} q_{1}\right\rfloor$ along the first dimension of the tensor and $\left\lfloor r_{d} q_{2}\right\rfloor$ along the second dimension, $r_{s} \in(0,1)$ is and hyperparameter which regulates it. Obviously the number of neurons, and consequently the number of outputs of this 2D-convolution layer, depends on all these parameters and is reported in Figure 14. Please refer to [23] for a more detailed treatment of the convolutional networks.

The 2D-convolution can be defined by a learned filter but also by a deterministic function like the maximum, the average, or the median. For example, in Figure 15 a 2D-max-pooling layer is reported, which is a 2Dconvolution layer where, instead of learning a filter, the authors just apply the maximum operator.

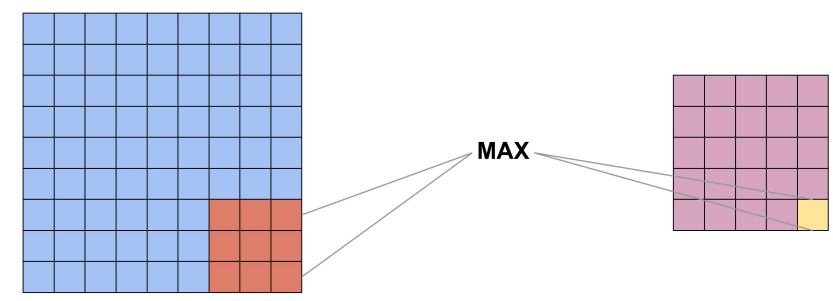

Figure 15: Max pooling on a two-dimensional tensor: substitution of the deterministic function max to the learned filter in a convolution on a two-dimensional tensor (see Figure 14. Note that, for simplicity, the padding, the dilation, and the stride have been not reported, since they are analogous to the ones of Figure 14.

Using the same principles described for the 2D-tensors (FS2, FS3, and FS5) it is possible to build a convolutional network for 3D-tensors (in this case FS4). The input space, in this case, will be a tensor of size $\mathbb{R}^{q_{1} \times q_{2} \times q_{3}}$ and, consequently, the filter size will be $\mathbb{R}^{\left\lfloor r_{f} q_{1}\right\rfloor_{\text {odd }} \times\left\lfloor r_{f} q_{2}\right\rfloor_{\text {odd }} \times\left\lfloor r_{f} q_{3}\right\rfloor_{\text {odd }} \text {. In }}$ an analogous way the other dimensions (padding, stride, and dilation) will change. An example of 3D-convolutional network is represented in Figure 16 . Analogously, the 3D-max-pooling can be defined.

At this point all the building blocks required to build the proposed architecture and DDMs for estimating the different NSPs based on the different 


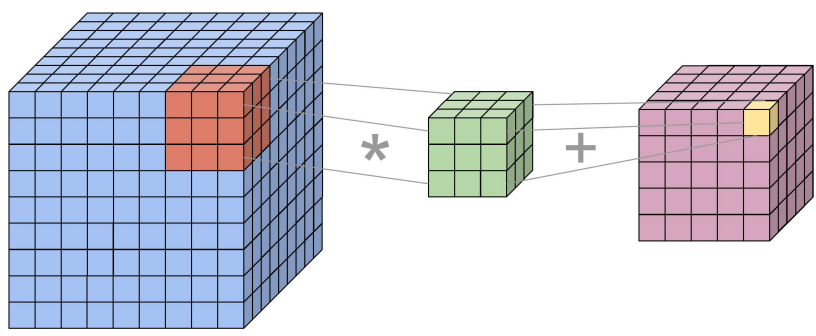

Figure 16: Convolution on a three-dimensional tensor (see Figure 14 for the meaning of the colors). Note that, for simplicity, the padding, the dilation, and the stride have been not reported, since they are analogous to the ones of Figure 14.

FSs are present. In particular, the authors defined a Dense Layer (Figure 11) with its different activation functions (e.g. RELU, Linear, Hyperbolic Tangent, etc.) and regularization (e.g. L2, Dropout, etc.), a Random Layer, namely a Dense Layer with random weights, the 2D and 3D Convolutional Layers (again with its activation functions), the 2D and 3D Max Pooling Layer. Since it will be required later, it has also been defined a Concatenation Layer, which simply takes in input whatever structure (e.g. scalars, vectors, or tensors) and reshapes everything in a large vector. The point is then how to combine them to get a suited architecture for the problem under exam.

The architecture of the proposed DDMs will be built incrementally to explain the different choices.

For what concerns the FS1, this FS is somehow analogous to the one of [19] and for this reason a simple SDDMs like the one of Figure 11 is enough. In order to limit the number of weights to be learned, the hidden layer will be a simple Random Layer (ELMs-style) with a hyperbolic tangent activation function to provide the necessary non-linearity, and the output layer will be a simple Dense Layer with linear activation function and the L2 regularisation to limit the overfitting. This structure basically emulates, with much fewer parameters to tune, the one proposed in [19]. The hyperparameter to be tuned are just the number of neurons of the hidden layer $n_{\mathrm{RL}} \in 2^{\{2,4,6,8,10\}}$ and the amount of regularisation defined by $\lambda \in 10^{\{-4.0,-3.5, \cdots,+3.0\}}$ (see Eq. (17)) in the output layer since the number of inputs is defined by FS1 and the number of output neurons is defined by the particular NSP to be predicted. The initialisation of the output Dense Layer is a simple zero-valued initialisation.

For what concerns instead FS2, FS3, FS4, and FS5, the process is a bit more complicated. The authors will first present the proposed method for 
dealing with the 2D-tensors (FS2, FS3, and FS5) and the treatment of the 3D-tensor (FS4) will be just summarised because analogous. As already mentioned before, the 2D-tensors cannot be simply stacked with FS1 by means of a Concatenation Layer and fed to the architecture of Figure 11 (see results of this approach in Section 4.2). For this reason, a more condensed representation of these FSs needs to be learnt, and, for this purpose, the convolution layers is the best choice. The only problem of the Convolutional Layers is that, based on the setting of their parameters, they are designed to react to just a particular scale of dimension and for this reason it would be good to have more layers which react to different scales. The solution that has been adopted in this paper is to use and Inception Layer [68] composed of three parallel Convolutional Layers (equipped with linear activation functions to mitigate the gradient vanishing effect, that will be clarified later, and no regularisation because of the intrinsic sparsity of the architecture) reacting to different scales. In order to limit the number of weights to be learned, one of the three Convolutional Layers is a simple 2D Max Pooling Layer. Then, in order to agglomerate all the information at different scales and produce a condensed representation, the outputs of the two 2D Convolutional and the 2D Max Pooling Layers are combined adopting a Concatenation Layer and then exploiting a Dense Layer (equipped with linear activation functions, again to mitigate the gradient vanishing effect, and dropout as regulariser). This building block is depicted in Figure 17. The architecture

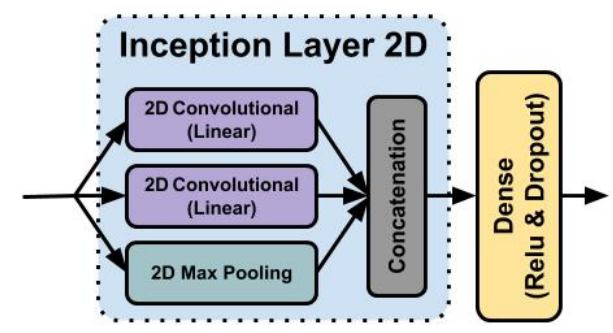

Figure 17: Proposed architecture for extracting a good representation from the twodimensional tensors (see FS2, FS3, and FS5 in Table 44 in the dataset described in Section 2 .

has multiple hyperparameters that have to be tuned. For the padding, a zero padding has been exploited. It is now necessary to tune for the $2 \mathrm{D}$ Convolutional and the 2D Max Pooling Layers the $r_{f}\left(r_{f}^{\mathrm{C}^{2} \mathrm{D}_{1}}, r_{f}^{\mathrm{C} 2 \mathrm{D}_{2}}, r_{f}^{\mathrm{MP} 2 \mathrm{D}_{1}} \in\right.$ $\{0.1,0.2,0.4\})$, the $r_{s}\left(r_{s}^{\mathrm{C} 2 \mathrm{D}_{1}}, r_{s}^{\mathrm{C} 2 \mathrm{D}_{2}}, r_{s}^{\mathrm{MP} 2 \mathrm{D}_{1}} \in\{0.1,0.2,0.4\}\right)$, and the $r_{d}$ $\left(r_{d}^{\mathrm{C} 2 \mathrm{D}_{1}}, r_{d}^{\mathrm{C} 2 \mathrm{D}_{2}}, r_{d}^{\mathrm{MP} 2 \mathrm{D}_{1}} \in\{0.1,0.2,0.4\}\right)$. Then, for the dense layer, it is necessary to tune the number of neurons $n_{\mathrm{DL}} \in 2^{\{2,4,6,8,10\}}$ and the dropout rate 
$r_{d} \in 10^{\{-3,-4,-2,-1\}}$, namely the number of neurons to randomly deactivate during training [23]. The problem of this architecture is its initialisation phase since a deterministic or random initialisation would not be sufficient to guarantee good performances [23]. For this reason the authors initialise, or more precisely pre-trained, the architecture of Figure 17 with a surrogate problem, using the autoencoders approach [23]. Basically, since the output of the dense layer in Figure 17 should be a good and condensed representation of the FSs (FS2, FS3, and FS5), based on that representation it should be possible to retrieve the original FSs. Subsequently, the weights have been initialised using the approach proposed in 69, the authors attach to the Dense Layer of Figure 17 another Dense Layer where the outputs are the same FS provided to the block as input, and finally the network is trained using the algorithms that will be explained later in this section. The architecture of the autoencoder for pre-training the block of Figure 17 is depicted in Figure 18. After this pre-training phase the last Dense Layer added for

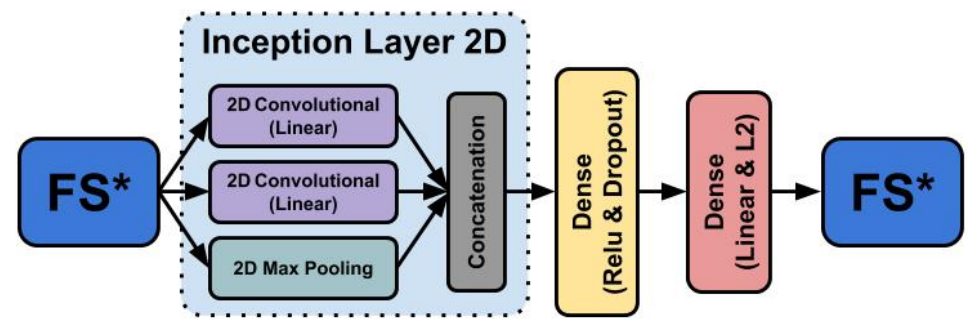

Figure 18: Architecture of the autoencoder for initialising the architecture presented in Figure 17.

the pre-training is removed, and the Inception Layer plus the dense layer after that have been kept.

The extension of this 2D block defined for FS2, FS3, and FS5 can be trivially extended to the case of FS4 where a 3D block needs to be developed.

At this point, it is possible to combine all outputs of the blocks developed for FS2, FS3, FS4, and FS5 in a Concatenation Layer together with FS1 and fed them to the same SDDMs described for FS1. It is possible to do perform this action since FS1 plus the outputs of the blocks developed for FS2, FS3, FS4, and FS5 is an informative and condensed information about all the features. The resulting architecture, namely the proposed DDM, is depicted in Figure 19.

What still needs to be described is how the network has been trained (or pre-trained the blocks just described for FS2, FS3, FS4, and FS5). As described before, many gradient descend-based algorithms [23] (e.g. SGD, 


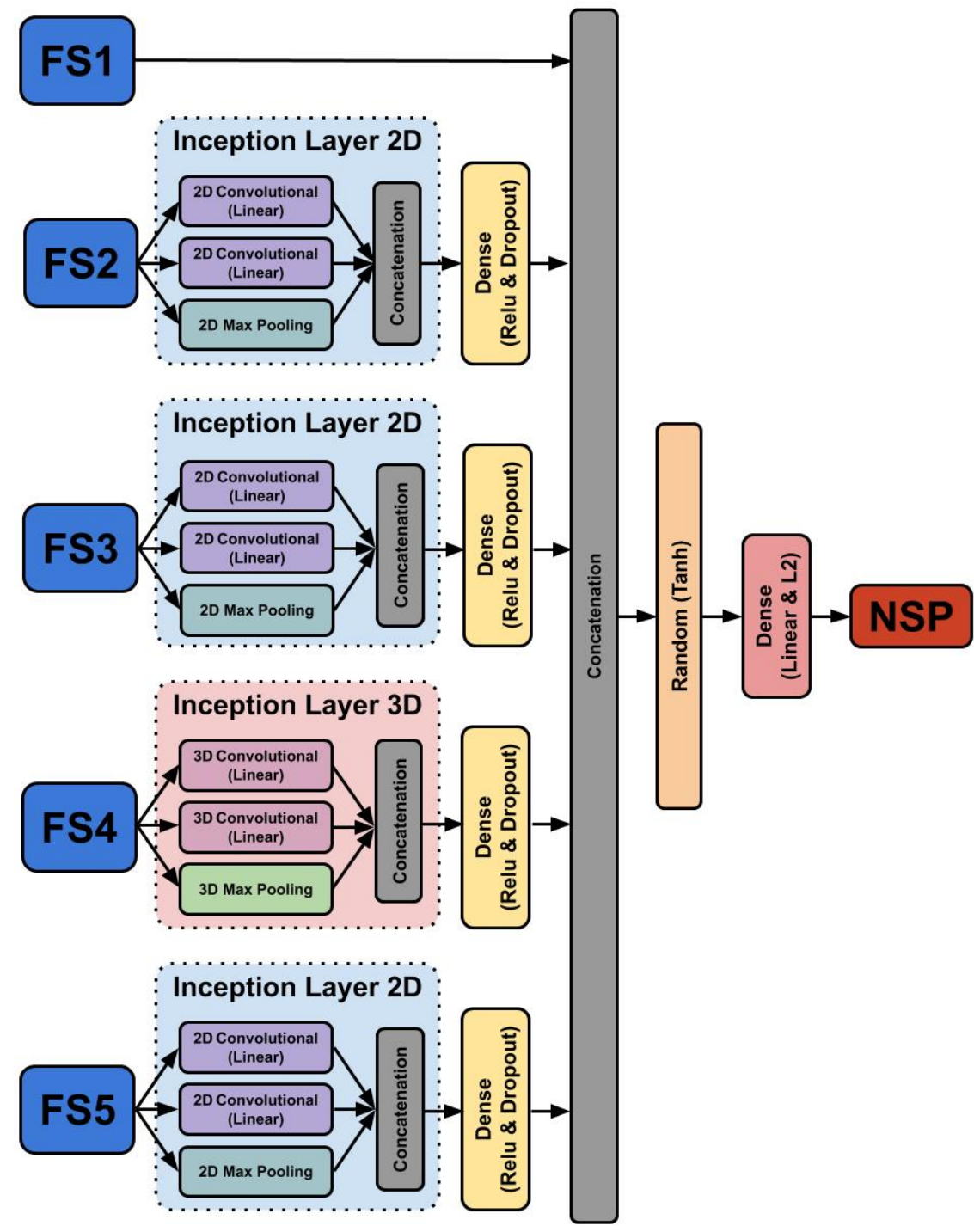

Figure 19: Proposed DDM architecture.

RMSprop, Adagrad, Adadelta, Adam, etc.) exist for solving the problem. The only issue of these algorithms is the Gradient Vanishing effect [23, namely the fact that in deep network the gradient tends to go to zero exponentially in the number of layers. For this reason, in the proposed architecture, the authors have exploited, in all the trained layers, linear or RELU activation functions which mitigate this problem [23]. Then, the 
Mini Batch Stochastic Gradient Descent (SGD) algorithm has been used, characterised by three hyperparameters [23]: learning rate of the gradient, momentum that accelerates SGD in the relevant direction, and batch-size of each iteration.

The last problem that authors need to solve is how to tune the hyperparameters of the proposed architecture. Since all DDMs are characterised by a set of hyperparameters $\mathcal{H}$ influencing their ability to estimate $\mu$, a proper Model Selection (MS) procedure, namely the process of tuning them to achieve optimal performances, needs to be performed [59]. Several methods exist for MS purpose but resampling methods, like the well-known $k$-Fold Cross Validation (KCV) or the nonparametric Bootstrap (BTS) approaches represent the state-of-the-art MS approaches when targeting real-world applications [59]. Resampling methods rely on a simple idea: the original dataset $\mathcal{D}_{n}$ is resampled once or many $(s)$ times, with or without replacement, to build two independent datasets called training, and validation sets, respectively $\mathcal{L}_{l}^{i}$ and $\mathcal{V}_{v}^{s}$, with $i \in\{1, \cdots, s\}$. Note that $\mathcal{L}_{l}^{i} \cap \mathcal{V}_{v}^{i}=\oslash$ and $\mathcal{L}_{l}^{i} \cup \mathcal{V}_{v}^{i}=\mathcal{D}_{n}$. Then, in order to select the best combination the hyperparameters $\mathcal{H}$ in a set of possible ones $\mathfrak{H}=\left\{\mathcal{H}_{1}, \mathcal{H}_{2}, \cdots\right\}$ for the proposed architecture or, in other words, to perform the MS phase, the following procedure has to be applied

$$
\mathcal{H}^{*}: \arg \min _{\mathcal{H} \in \mathfrak{H}} \frac{1}{s} \sum_{i=1}^{s} \hat{\mathrm{L}}\left(h_{\left\{\mathcal{H}, \mathcal{L}_{l}^{i}\right\}}^{*}, \mathcal{V}_{v}^{i}\right)
$$

where $h_{\left\{\mathcal{H}, \mathcal{L}_{l}^{i}\right\}}^{*}$ is the model with its set of hyperparameters $\mathcal{H}$ learned with with the data $\mathcal{L}_{l}^{i}$. Since the data in $\mathcal{L}_{l}^{i}$ are independent of the ones in $\mathcal{V}_{v}^{i}$, the idea is that $\mathcal{H}^{*}$ should be the set of hyperparameters which allows achieving a small error on a data set that is independent of the training set. In this work, the authors will exploit the BTS procedure and consequently $s=100$, if $l=n$ and the resampling must be done with replacement [59].

\subsection{Hybrid Models (HMs)}

The problem that authors want to address here is how to construct a model able to both take into account the physical knowledge about the problem encapsulated in the PMs of Section 3.2 and the information hidden in the available data described in Section 2 as the DDMs of Section 3.3. For this reason the proposed HM is a combination of the PMs and the DDMs.

In order to reach this goal different approaches exist (see e.g. [19, 70, 71]) but all these methods have been developed in the context of conventional DDMs (like the KLRS mentioned above) and not for advance DDMs (es 
the one based on DNNs described in Section 3.3). In fact, for conventional DDMs there are many ways of including the knowledge encapsulated in the PMs. For example, in [70, 71] authors simply add to the input space of the DDMs the prediction of the PMs, while in [19, 71] authors tried to build a model able to contemporary learn the target task and how the PMs behave.

These different flavours of HMs, for conventional DDMs, are due to the fact that the model functional form for conventional DDMs cannot be arbitrary modified without compromising their ability to effectively and efficiently learn from data or weakening they theoretical properties [23, 56]. Vice versa, the architecture of the advanced DDMs based on DNNs described in Section 3.3 can be easily and almost arbitrary modified to meet the requirements of the particular application. Moreover, different ways of changing the architecture may results in the same effect because of the nature of the functional form of these DDMs, and for this reason the simplest solution can be chosen.

For example, in the case under examination, it could be possible to change the architecture of the proposed DDM depicted in Figure 19 using the two main different philosophies introduced in [19, 70, 71] which consists in

I changing the FS, namely the input space, or

II force the DDMs to learn contemporary the NSPs and the PMs, namely change the output space.

For what concerns the Option II the modification is trivial while the Option II is not as much trivial as it may seem since it is required to define where and how the prediction of the PMs should be fed to the DDM. Since the PMs already provide a good approximation of the propeller characteristic, in this particular case an actual NSP approximation, the most natural choice would be to consider this information at the same level of the FS1 that need to be fed to the layer which condensates all the information about the different FSs in order to improve its representativity. But such a choice is somehow equivalent to change the output space of the DDM since this would result in a consistent change of the last layers of the DDMs (in particular the expressivity, of size, of the random layer) [23. Since these two modifications, in the proposed DDM, would have a similar effect, the authors decide to use the Option I since it affects more directly the last layers, not influencing the other ones. The result is the HM architecture depicted in Figure 20, where the authors just underline, for simplicity, the differences between the DDMs of Figure 19 and the proposed HM.

Note that the HMs can be built just for the NSP for which a PM is provided, able to estimate all, or just a subset, of the parameters of the 


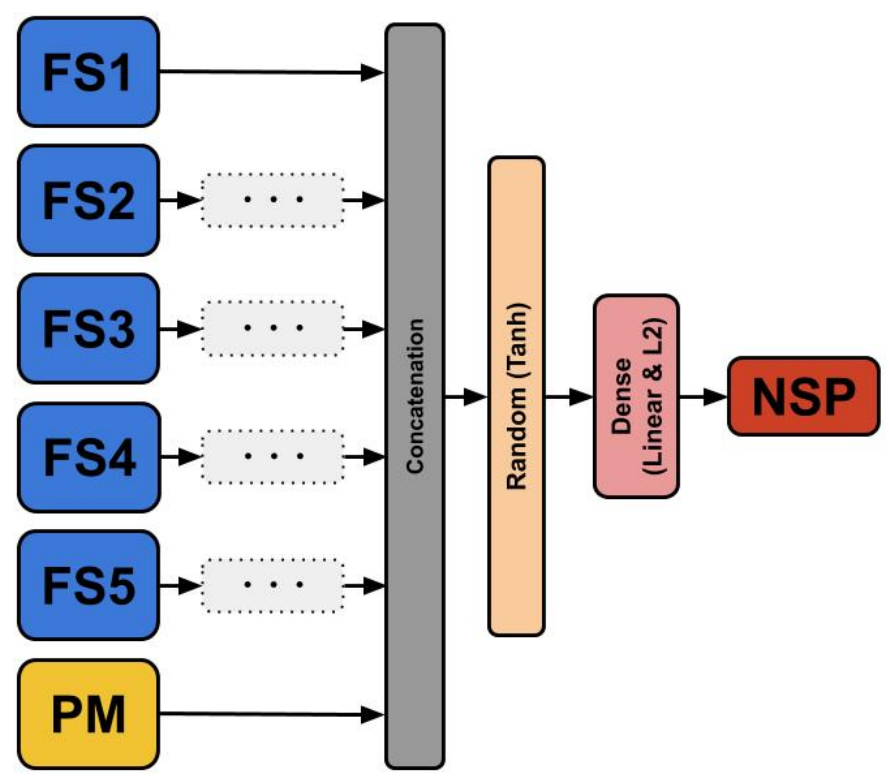

Figure 20: DNN-based HM architecture (see Figure 19 for the missing pieces).

NSP. Hence, in this case, the HM can be defined just for all the NSPs (see Table 4).

Note also that the hyperparameters of the architecture, also for the HMs, need to be tuned with the same procedure described in Section 3.3 for the DDMs.

\section{Experimental Results}

In this section, the performances of the PMs, DDMs, and HMs, (see Sections 3.2, 3.3, and 3.4 are tested and compared by means of the data described in Section 2 and the performance measures described in Section 3.1. Two different scenarios are considered, analogously to what presented in [19].

For what concerns the PM, please recall that PMs are just able to predict a subset of the parameters of the different NSPs, while the DDMs are able to predict all the targets. HMs differ from the DDMs every time the PMs are able to predict the spectral parametrization or a part of it. The set of hyperparameters tuned during the MS phase are the same as those of the DDMs.

All the tests have been repeated 30 times and the average results are reported, together with their t-student $95 \%$ confidence interval, in order to ensure the statistical consistency of the results. 


\subsection{Scenarios}

Two modelisation scenarios have been studied

- Interpolation Scenario: in this case the models try to predict the propeller noise spectra main characteristics in various, but different, loading conditions within the ones exploited for building the model;

- Extrapolation Scenario: in this scenario, the models try to predict the propeller noise in groups of loading conditions characterised by different cavitation extents with respect to those exploited for building the model. These groups of loading conditions are defined based on the combination of thrust coefficient and cavitation number, as exemplified in Figure 21 on a typical cavitation bucket: the cavitation extent grows from the upper left corner of the plot (group G2) to the lower right corner (group G6).

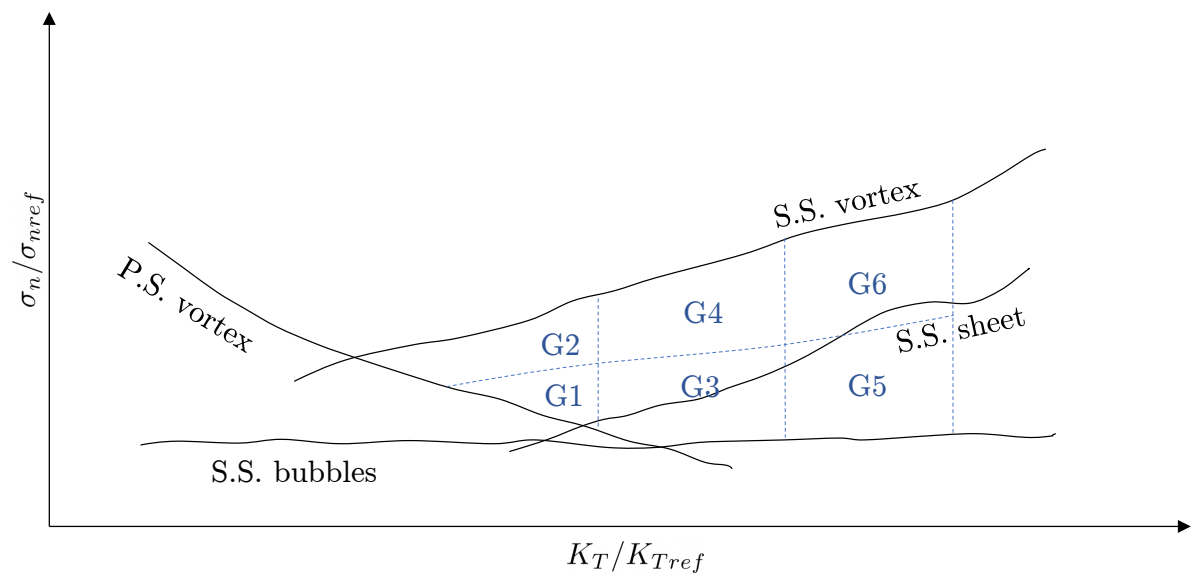

Figure 21: Extrapolation groups.

Basically the two scenarios just differ in the definitions of $\mathcal{D}_{n}$ and $\mathcal{T}_{m}$, that are the subset of data exploited for building and testing the models.

The interpolation case is the simplest one. In this scenario $\mathcal{D}_{n}$ and $\mathcal{T}_{m}$ have been created by splitting randomly all the samples of the datasets described in Section 2 keeping $90 \%$ of the data in $\mathcal{D}_{n}$ and the remaining $10 \%$ in $\mathcal{T}_{m}$. In this way the models have been tested in their ability to predict the propeller noise spectra main characteristics in various, but different, loading conditions within those exploited for building the models.

The extrapolation scenario tests the capability of the models to predict radiated noise for cases not included in the variable domain of the data used 
to build them. The practical application of this scenario is the prediction of noise for those full scale propeller operational conditions which cannot be consistently reproduced at model scale due to viscous scale effects, as explained in [19]. In order to obtain an indication of the extrapolation performance, the authors included in $\mathcal{D}_{n}$ samples of only five of the six groups of different operational conditions and use the sixth group as $\mathcal{T}_{m}$.

\section{2. $C D D M s$ vs $A D D M s$}

In order to compare the CDDMs (like the KRLS-based DDMs introduced in [19] and recalled in Section 3.3 and the ADDMs proposed in this work, the authors have first reported in Table 5 the errors measured with the MAPE in the interpolation and extrapolation scenarios with different FSs for the different NSPs.

From the results reported in Table 5, the CDDMs show comparable performances to ADDMs only when the FS1 is considered alone, hence when the cardinality of the FS is not too high with respect to the number of samples. If multidimensional features are considered, the error for CDDMs is at least doubled with respect to ADDMs. The worst performances for the CDDMs are attained for FS1+FS4 (FS4 is the single feature with the higher cardinality) and for FSAll. The lowest error for the CDDMs, when multidimensional features are considered, is in the case FS1+FS5 probably because FS5 (the blade circulation) account for important information on blade loading with smaller dimensions than FS4. Conversely, the minimum prediction error is obtained, for the ADDMs, when all the features are considered. Observed errors are lower than $5 \%$ in the interpolation scenario and lower than $9 \%$ in the extrapolation scenario.

In summary, results suggest that only by using the ADDMs it is possible to improve the performance of the model exploiting more rich and complex features, and the relative improvement can be larger than $50 \%$. On the other hand, it must be noted that the absolute errors obtained with the CDDMs and the simplest feature set FS1 are only a few percentages higher than the minimum errors obtained with the ADDMs. Notwithstanding this, the improvements achieved exploiting the ADDMs with more complete and physically meaningful features are significant.

\section{3. $P M s$ vs $D D M s$ vs $H M s$}

From now on, for the sake of simplicity, the proposed ADDMs will be referred to as DDMs since, as reported in Section 4.2, the ADDMs are the most effective ones for the scopes of this paper. In order to compare the PMs, DDMs, and HMs Table 6 reports the errors measured with the MAPE 
in the interpolation and extrapolation scenarios with different FSs for the different NSPs. Note that the PMs are able to fully predict only the NSP1 and it always uses only FS0. Instead, since the PMs is able to predict part of all the NSPs it is always possible to build the HMs.

The results highlight the limits of the PMs, which are not able to accurately predict all the trends present in the experimental data. Nevertheless, the information enclosed in these simple formulations allows to improve the performance of DDMs significantly, as confirmed by the results obtained for the HMs. The improvement with respect to the simple DDMs is more significant when all the features are considered and, in particular, in the extrapolation scenario, where the MAPE for the HMs is about $2 \%$ lower than the DDMs. This result agrees with the higher capabilities of HMs to generalise, thanks to the information included in the PMs.

\subsection{The Effect of Using the Different FSs on the DDMs and the HMs}

From the results reported in Table 6, it is also possible to understand the effect of using the different FSs on the DDMs and the HMs.

The DDMs seem to be able to well exploit these multidimensional inputs. When one multidimensional feature (FS2, FS3, FS4, FS5) is added to FS1, results are generally improved. Surprisingly, the effects of the different multidimensional features are all rather similar, preventing to rank these features based on their importance. The best performances are achieved by the DDMs, when all the possible features are considered. However, the absolute improvement with respect to the use of one single multidimensional feature added to FS1 is not large given the low errors.

The same patterns are reflected in the HMs (Table 6). Nevertheless, the HMs seem to be even less influenced by the different combinations of FS1 and multidimensional features. If all the features are considered, the HMs show the best performances among all the considered models.

\subsection{The Effect of Using Different NSPs}

Table 6 also allows us to understand the effect of the different target definitions based on the models' performances in predicting them, the same order is obtained for PMs, DDMs, and HMs. In particular, going from the best case to the worst, the order is: NSP1, NSP4 and NSP5 (similar outcome), NSP2 and lastly NSP3. However, the differences in performances are rather small, meaning that the choice of the spectral representation does not affect significantly the predictive performances of the models.

The lowest error observed for NSP1 was reasonably expected. Actually, this target has a strong physical meaning and usually shows clear trends 
among the dataset. In fact, NSP1 is composed by a single point instead of a complete spectrum, therefore comparing it with the other target definitions may be questionable. It is less trivial to explain the good performances observed in the prediction of the whole one-third octave spectrum (NSP4 and NSP5). This result is remarkable because the one-third octave spectra are a representation of the noise recognised in the acoustic field. Moreover, its definition is easier than the simplified spectrum, being less prone to human error or susceptible to outliers. Therefore its use may simplify the dataset building phase.

A ML tool able to reproduce accurately both the NSP4, NSP5 and the center peak (NSP1) represents an optimal solution for the task at hand. The use of spectral slopes in NSP3 was an attempt to overcome the issues related to the complex trends featured by the breakpoints. These results demonstrate that this modification to the description of the simplified spectrum does not provide any further improvements of the model capabilities. Actually, the errors for NSP3 are slightly larger than for NSP2 yet acceptable.

\subsection{The Best PMs, DDMs, and HMs}

In order to better detail the quality of the best PMs, DDMs, and HMs in predicting the different parameters of the different NSPs, the errors measured with the MAE, MAPE, and PPMCC in the interpolation and extrapolation scenarios, with the best FS according to Table 6 are reported in Tables 7. 11. The word "best" is used to intend the model which produces the best accuracy, or lower error, between the ones which use different FSs according to Table 6 .

The vortex peak (frequency and level) is present in NSP1, NSP2 and NSP3 and the errors for these targets are reported in Tables 7 . 9, in terms of MAE, MAPE and PPMCC. The best interpolation MAE for $\mathrm{f}_{\mathrm{c}}$ is below $60 \mathrm{~Hz}$ and for $\mathrm{RNL}_{\mathrm{c}}$ it is lower than $1 \mathrm{~dB}$, both for DDMs and HMs. In extrapolation the accuracy slightly decreases but it is still remarkable. In Figure 22 the comparison reported shows the PM, and the best DDM and HM predicting the NSP1, for the interpolation scenario. Looking more in detail, it can be noticed that the DDMs/HMs are able to predict cases in which the PM fails, i.e. when FS0 is not sufficient to characterise the samples. In the extrapolation (Figure 23) the samples are more scattered, hence the accuracy is lower but still satisfactory. In both cases, some outliers are visible in the DDMs and HMs: these samples should be investigated and properly treated, e.g. adjusting the position of the peak or removing them from the dataset. 
The spectrum level at $100 \mathrm{kHz}\left(\mathrm{RNL}_{\mathrm{b}}\right)$ is present in NSP2 and NSP3 (Tables 8- 9p). The MAE is lower than $1 \mathrm{~dB}$ both in interpolation and extrapolation. Similar to the vortex peak, this target is easy to be predicted because its value changes rather smoothly depending on propeller loading, hence the current features are able to fully characterise its behaviour.

The NPS2 is the best predicted spectrum simplification if it is considered as a whole (Table 6). A detailed view of the estimation error is reported in Table 8 for each target composing NSP2. In the prediction of the breakpoints frequencies and level, the MAPE is almost identical between breakpoint 1 and 2, both for interpolation and extrapolation meaning that the absolute error is proportional to the absolute value of the targets. Indeed, the best MAPE for $\mathrm{f}_{\mathrm{bp} 1}$ and for $\mathrm{f}_{\mathrm{bp} 2}$ is 3.0 (HM, interpolation), but the correspondent MAE are $27.6 \mathrm{~Hz}$ and $2067 \mathrm{~Hz}$. Therefore, $\mathrm{f}_{\mathrm{bp} 2}$ still suffer from worse prediction compared to $\mathrm{f}_{\mathrm{bp} 1}$, because of some outliers due to the inherent physical phenomenon. The same tendency is visible for the levels of the two breakpoints but being the decibel a logarithmic quantity, the MAE is somewhat more contained.

In order to overcome the inherent weakness in the breakpoints detection, and improve the accuracy in the simplified spectrum prediction, the slopes of the different parts composing the simplified spectrum have been considered as an alternative target. They are expressed as $\mathrm{dB} /$ oct, hence they represent the change in $\mathrm{dB}$ corresponding to a doubling in frequency. In Table 9 the accuracy in the NSP3 prediction is reported. It is evident that $\gamma$ has a MAE of one order of magnitude inferior to $\alpha$ and $\beta$. This can be attributed to the higher stability (with respect to the WPs) of this portion of the spectrum, and to the aforementioned ease in the detection of one of the extreme points on which it is calculated $\left(100 \mathrm{kHz}, \mathrm{RNL}_{\mathrm{b}}\right)$.

Conversely, the two other slopes still suffer from the same problem discussed for the breakpoints, even if the overall prediction accuracy is remarkable.

The NSP4 (noise corrected by means of transfer functions) and the NSP5 (noise corrected for spherical propagation) are visible in Tables 10 and 11 . respectively. Each column refers to a one-third octave band (from 1 to 24 ) in the range $0.4 \mathrm{kHz}$ to $80 \mathrm{kHz}$ for the $\mathrm{DDM} / \mathrm{HM}$, instead the $\mathrm{PM}$ is available only for the range $1 \mathrm{kHz}$ to $80 \mathrm{kHz}$. The different levels are well predicted, both in interpolation and extrapolation, no particular trends seem to exist among the different parts of the spectrum. 


\begin{tabular}{|c|c|c|c|c|c|c|c|c|c|c|c|}
\hline \multirow{2}{*}{\multicolumn{2}{|c|}{$\mathrm{F}$}} & \multicolumn{2}{|c|}{ NSP1 } & \multicolumn{2}{|c|}{ NSP2 } & \multicolumn{2}{|c|}{ NSP3 } & \multicolumn{2}{|c|}{ NSP4 } & \multicolumn{2}{|c|}{ NSP5 } \\
\hline & & CDDM & ADDM & CDDM & ADDM & CDDM & ADDM & $\mathrm{DM}$ & ADDM & DDM & $\mathrm{ADDM}$ \\
\hline \multirow{6}{*}{. } & 1 & $4.2 \pm 0.2$ & $4.4 \pm 0.3$ & $5.5 \pm 0.3$ & $5.5 \pm 0.3$ & $6.4 \pm 0.3$ & $6.4 \pm 0.3$ & $4.5 \pm 0.2$ & $4.5 \pm 0.2$ & $4.5 \pm 0.3$ & $4.5 \pm 0.3$ \\
\hline & 1,2 & $9.0 \pm 0.3$ & $3.5 \pm 0.2$ & $10.4 \pm 0.3$ & $4.6 \pm 0.3$ & $11.2 \pm 0.3$ & $5.5 \pm 0.3$ & $8.3 \pm 0.3$ & $3.8 \pm 0.2$ & $8.8 \pm 0.3$ & $3.7 \pm 0.2$ \\
\hline & 1,3 & $8.9 \pm 0.3$ & $3.3 \pm 0.2$ & $10.6 \pm 0.3$ & $4.7 \pm 0.3$ & $11.3 \pm 0.3$ & $5.5 \pm 0.3$ & $8.3 \pm 0.3$ & $3.8 \pm 0.2$ & $8.8 \pm 0.3$ & $3.7 \pm 0.2$ \\
\hline & 1,4 & $12.1 \pm 0.3$ & $3.5 \pm 0.2$ & $13.5 \pm 0.3$ & $4.6 \pm 0.3$ & $14.3 \pm 0.3$ & $5.6 \pm 0.3$ & $10.5 \pm 0.3$ & $3.9 \pm 0.2$ & $11.4 \pm 0.3$ & $3.7 \pm 0.2$ \\
\hline & 1,5 & $7.3 \pm 0.3$ & $3.7 \pm 0.2$ & $8.5 \pm 0.3$ & $4.8 \pm 0.3$ & $9.5 \pm 0.3$ & $5.5 \pm 0.3$ & $6.8 \pm 0.3$ & $3.8 \pm 0.2$ & $7.0 \pm 0.3$ & $3.7 \pm 0.2$ \\
\hline & All & $14.8 \pm 0.3$ & $2.8 \pm 0.2$ & $16.4 \pm 0.3$ & $3.9 \pm 0.2$ & $17.4 \pm 0.3$ & $4.7 \pm 0.3$ & $12.8 \pm 0.3$ & $3.2 \pm 0.2$ & $14.0 \pm 0.3$ & $3.0 \pm 0.2$ \\
\hline \multirow{6}{*}{ 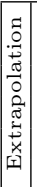 } & 1 & $8.0 \pm 0.3$ & $8.0 \pm 0.3$ & $9.3 \pm 0.3$ & $9.5 \pm 0.3$ & $10.6 \pm 0.3$ & $10.3 \pm 0.3$ & $7.5 \pm 0.3$ & $7.5 \pm 0.3$ & $7.9 \pm 0.3$ & $7.9 \pm 0.3$ \\
\hline & 1,2 & $13.0 \pm 0.3$ & $6.9 \pm 0.3$ & $14.5 \pm 0.3$ & $8.4 \pm 0.3$ & $15.3 \pm 0.3$ & $9.4 \pm 0.3$ & $11.2 \pm 0.3$ & $6.8 \pm 0.3$ & $12.2 \pm 0.3$ & $7.0 \pm 0.3$ \\
\hline & 1,3 & $12.9 \pm 0.3$ & $7.1 \pm 0.3$ & $14.5 \pm 0.3$ & $8.5 \pm 0.3$ & $15.3 \pm 0.3$ & $9.4 \pm 0.3$ & $11.3 \pm 0.3$ & $6.7 \pm 0.2$ & $12.2 \pm 0.3$ & $7.0 \pm 0.3$ \\
\hline & 1,4 & $16.1 \pm 0.3$ & $7.2 \pm 0.3$ & $17.4 \pm 0.3$ & $8.5 \pm 0.3$ & $18.4 \pm 0.3$ & $9.3 \pm 0.3$ & $13.5 \pm 0.3$ & $6.8 \pm 0.2$ & $14.8 \pm 0.3$ & $7.0 \pm 0.3$ \\
\hline & 1,5 & $10.9 \pm 0.3$ & $6.9 \pm 0.3$ & $12.5 \pm 0.3$ & $8.5 \pm 0.3$ & $13.4 \pm 0.3$ & $9.2 \pm 0.3$ & $9.8 \pm 0.3$ & $6.8 \pm 0.3$ & $10.5 \pm 0.3$ & $7.0 \pm 0.3$ \\
\hline & All & $19.0 \pm 0.3$ & $6.0 \pm 0.3$ & $20.4 \pm 0.3$ & $7.4 \pm 0.3$ & $21.4 \pm 0.3$ & $8.3 \pm 0.3$ & $15.8 \pm 0.3$ & $6.1 \pm 0.2$ & $17.5 \pm 0.4$ & $6.1 \pm 0.3$ \\
\hline
\end{tabular}

Table 5: Comparison between CDDMs proposed in [19] and the proposed ADDM. Table reports the errors measured with the MAPE (see Subsection 3.1) in the interpolation and extrapolation scenarios with different FSs for the different NSPs.

\subsection{Interpolation vs Extrapolation}

Tables 6-11 also allows us to understand the behaviour of the different models in the interpolation and extrapolation scenarios. The models here presented show promising results both in the interpolation and extrapolation scenario. Indeed, the error in extrapolation are doubled respect to the interpolation case, but being the latter really small, also the performances in extrapolation are widely acceptable. In extrapolation, the MAPE for the DDMs and the HMs is lower than $10 \%$.

\section{Conclusions}

In this paper, an approach to estimate propeller cavitation noise spectrum by means of numerical models has been presented. In order to verify a propeller to be compliant with noise requirements only considering the information available at the design stage, the proposed models have been developed exploiting a dataset collected through model scale measurements in a cavitation tunnel, combined with the detailed flow characterisation obtained by Boundary Element Method calculations. As a result, different feature sets have been considered with different levels of complexity, ranging from simple propeller design parameters to quantities describing the flow on propeller blades in details, together with different target sets, in order to asses the model performance on different representations of cavitation noise. Three main strategies were considered in order to create a predictive model able to define a relationship between each combination of the input sets with the output sets. Physical models, relying on equations describing 


\begin{tabular}{|c|c|c|c|c|c|c|c|c|c|c|c|c|}
\hline \multirow{2}{*}{\multicolumn{2}{|c|}{$\mathrm{FS}$}} & \multicolumn{3}{|c|}{ NSP1 } & \multicolumn{2}{|c|}{ NSP2 } & \multicolumn{2}{|c|}{ NSP3 } & \multicolumn{2}{|c|}{ NSP4 } & \multicolumn{2}{|c|}{ NSP5 } \\
\hline & & PM & DDM & $\mathrm{HM}$ & DDM & HM & DDM & HM & DDM & HM & DDM & $\mathrm{HM}$ \\
\hline \multirow{6}{*}{ 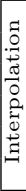 } & 1 & \multirow{6}{*}{$11.6 \pm 1.0$} & $4.4 \pm 0.3$ & $4.3 \pm 0.3$ & $5.5 \pm 0.3$ & $5.6 \pm 0.3$ & $6.4 \pm 0.3$ & $6.3 \pm 0.3$ & $4.5 \pm 0.2$ & $4.5 \pm 0.2$ & $4.5 \pm 0.3$ & $4.5 \pm 0.3$ \\
\hline & 1,2 & & $3.5 \pm 0.2$ & $2.8 \pm 0.2$ & $4.6 \pm 0.3$ & $3.9 \pm 0.3$ & $5.5 \pm 0.3$ & $4.7 \pm 0.3$ & $3.8 \pm 0.2$ & $3.2 \pm 0.2$ & $3.7 \pm 0.2$ & $3.0 \pm 0.2$ \\
\hline & 1,3 & & $3.3 \pm 0.2$ & $2.8 \pm 0.2$ & $4.7 \pm 0.3$ & $3.9 \pm 0.3$ & $5.5 \pm 0.3$ & $4.7 \pm 0.3$ & $3.8 \pm 0.2$ & $3.2 \pm 0.2$ & $3.7 \pm 0.2$ & $3.0 \pm 0.2$ \\
\hline & 1,4 & & $3.5 \pm 0.2$ & $2.9 \pm 0.2$ & $4.6 \pm 0.3$ & $3.9 \pm 0.2$ & $5.6 \pm 0.3$ & $4.7 \pm 0.3$ & $3.9 \pm 0.2$ & $3.2 \pm 0.2$ & $3.7 \pm 0.2$ & $3.1 \pm 0.2$ \\
\hline & 1,5 & & $3.7 \pm 0.2$ & $2.8 \pm 0.2$ & $4.8 \pm 0.3$ & $3.9 \pm 0.2$ & $5.5 \pm 0.3$ & $4.8 \pm 0.3$ & $3.8 \pm 0.2$ & $3.2 \pm 0.2$ & $3.7 \pm 0.2$ & $3.1 \pm 0.2$ \\
\hline & All & & $2.8 \pm 0.2$ & $2.4 \pm 0.2$ & \pm 0.2 & $2.7 \pm 0.2$ & $=0.3$ & $4 \pm 0.2$ & \pm 0.2 & $2.2 \pm 0.2$ & $3.0 \pm 0.2$ & $2.3 \pm 0.2$ \\
\hline \multirow{6}{*}{1} & 1 & \multirow{6}{*}{$12.1 \pm 1.1$} & $8.0 \pm 0.3$ & $8.0 \pm 0.3$ & $9.5 \pm 0.3$ & $9.4 \pm 0.3$ & $10.3 \pm 0.3$ & $10.4 \pm 0.3$ & $7.5 \pm 0.3$ & $7.5 \pm 0.3$ & $7.9 \pm 0.3$ & $7.9 \pm 0.3$ \\
\hline & 1,2 & & $6.9 \pm 0.3$ & $6.1 \pm 0.3$ & $8.4 \pm 0.3$ & $7.3 \pm 0.3$ & $9.4 \pm 0.3$ & $8.2 \pm 0.3$ & $6.8 \pm 0.3$ & $6.0 \pm 0.2$ & $7.0 \pm 0.3$ & $6.2 \pm 0.3$ \\
\hline & 1,3 & & $7.1 \pm 0.3$ & $5.8 \pm 0.3$ & $8.5 \pm 0.3$ & $7.6 \pm 0.3$ & $9.4 \pm 0.3$ & $8.5 \pm 0.3$ & $6.7 \pm 0.2$ & $6.0 \pm 0.2$ & $7.0 \pm 0.3$ & $6.2 \pm 0.3$ \\
\hline & 1,4 & & $7.2 \pm 0.3$ & $6.0 \pm 0.3$ & $8.5 \pm 0.3$ & $7.5 \pm 0.3$ & $9.3 \pm 0.3$ & $8.5 \pm 0.3$ & $6.8 \pm 0.2$ & $6.0 \pm 0.2$ & $7.0 \pm 0.3$ & $6.1 \pm 0.3$ \\
\hline & 1,5 & & $6.9 \pm 0.3$ & $5.8 \pm 0.3$ & $8.5 \pm 0.3$ & $7.4 \pm 0.3$ & $9.2 \pm 0.3$ & $8.3 \pm 0.3$ & $6.8 \pm 0.3$ & $6.0 \pm 0.2$ & $7.0 \pm 0.3$ & $6.1 \pm 0.3$ \\
\hline & All & & $6.0 \pm 0.3$ & $4.3 \pm 0.3$ & $7.4 \pm 0.3$ & $5.6 \pm 0.3$ & $8.3 \pm 0.3$ & $6.4 \pm 0.3$ & $6.1 \pm 0.2$ & $4.6 \pm 0.2$ & $6.1 \pm 0.3$ & $4.5 \pm 0.3$ \\
\hline
\end{tabular}

Table 6: Comparison between PMs, DDMs, and HMs. Table reports the errors measured with the MAPE (see Subsection 3.1) in the interpolation and extrapolation scenarios with different FSs for the different NSPs. Note that the PMs are only able to fully predict the NSP1 and they does not change if we change the FS since they always use just a subset of the FS1 (see Subsection 3.2. For the detailed accuracies of the PMs in predicting each parameters which is able to predict (see Table 4) see Tables 7, 8, 9, and 11. Instead, since the PMs is able to predict part of the NSP1, NSP2, NSP3, and NSP5 (see Subsection 3.2 we cab build the HMs for those NSPs (see Subsection 3.4.

\begin{tabular}{|c|c|c|c|c|c|}
\hline & & & FS* & $f_{\mathrm{c}}$ & $\mathrm{RNL}_{\mathrm{C}}$ \\
\hline \multirow{6}{*}{$\begin{array}{l}\text { 国 } \\
⿱ 亠 幺\end{array}$} & & $\mathrm{PM}$ & - & $202.0 \pm 21.5$ & $4.6 \pm 0.3$ \\
\hline & $\dot{\Xi}$ & DDM & all & $57.2 \pm 4.1$ & $1.0 \pm 0.1$ \\
\hline & & HM & all & $48.4 \pm 3.8$ & $0.8 \pm 0.1$ \\
\hline & & $\mathrm{PM}$ & 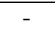 & $206.2 \pm 21.4$ & $4.9 \pm 0.4$ \\
\hline & 䓂 & DDM & all & $122.6 \pm 5.5$ & $2.1 \pm 0.1$ \\
\hline & & $\mathrm{HM}$ & all & $84.7 \pm 5.4$ & $1.5 \pm 0.1$ \\
\hline \multirow{6}{*}{ 䛼 } & & $\mathrm{PM}$ & - & $9.9 \pm 1.1$ & $13.2 \pm 1.0$ \\
\hline & $\stackrel{\vec{\Xi}}{\Xi}$ & DDM & all & $2.8 \pm 0.2$ & $2.9 \pm 0.2$ \\
\hline & & $\mathrm{HM}$ & all & $2.4 \pm 0.2$ & $2.4 \pm 0.2$ \\
\hline & & $\mathrm{PM}$ & 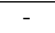 & $10.1 \pm 1.0$ & $14.1 \pm 1.1$ \\
\hline & 荋 & DDM & all & $6.0 \pm 0.3$ & $5.9 \pm 0.3$ \\
\hline & & $\mathrm{HM}$ & all & $4.1 \pm 0.3$ & $4.4 \pm 0.3$ \\
\hline \multirow{6}{*}{0} & \multirow{3}{*}{ 苞 } & $\mathrm{PM}$ & - & $0.76 \pm 0.01$ & $0.52 \pm 0.02$ \\
\hline & & $\mathrm{DDM}$ & all & $0.99 \pm 0.01$ & $0.99 \pm 0.01$ \\
\hline & & $\mathrm{HM}$ & all & $0.99 \pm 0.01$ & $0.99 \pm 0.01$ \\
\hline & \multirow{3}{*}{ 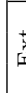 } & PM & - & $0.76 \pm 0.01$ & $0.39 \pm 0.12$ \\
\hline & & DDM & all & $0.99 \pm 0.01$ & $0.99 \pm 0.01$ \\
\hline & & $\mathrm{HM}$ & all & $0.99 \pm 0.01$ & $0.99 \pm 0.01$ \\
\hline
\end{tabular}

Table 7: Comparison between the best PM, DDM, and HM in predicting the different parameters of NSP1 according to Table 6. Table reports the errors measured with the MAE, MAPE, and PPMCC (see Subsection 3.1) in the interpolation and extrapolation scenarios with best FS according to Table 6 for the different parameters of NSP1 (see Table 4). Note that for the PM the best FS is not indicated since it always use just a subset of the FS1 (see Subsection 3.2. 


\begin{tabular}{|c|c|c|c|c|c|c|c|c|c|c|}
\hline & & & FS & $f_{\mathrm{C}}$ & $\mathrm{RNL}_{\mathrm{c}}$ & $\mathrm{RNL}_{\mathrm{b}}$ & $f_{\mathrm{bp} 1}$ & $f_{\mathrm{bp} 2}$ & $\mathrm{RNL}_{\mathrm{bp} 1}$ & $\mathrm{RNL}_{\mathrm{bp} 2}$ \\
\hline \multirow{6}{*}{$\underset{\Sigma}{\mid}$} & & PM & - & $202.0 \pm 21.5$ & $4.6 \pm 0.3$ & $14.3 \pm 1.5$ & - & - & - & - \\
\hline & $\stackrel{\rightleftarrows}{\Xi}$ & DDM & all & $58.7 \pm 4.3$ & $1.0 \pm 0.1$ & $1.6 \pm 0.1$ & $39.3 \pm 2.4$ & $2757.9 \pm 182.1$ & $1.4 \pm 0.1$ & $2.4 \pm 0.1$ \\
\hline & & $\mathrm{HM}$ & all & $48.9 \pm 3.9$ & $0.8 \pm 0.1$ & $1.0 \pm 0.1$ & $27.6 \pm 2.0$ & $2067.0 \pm 146.6$ & $0.9 \pm 0.1$ & $1.5 \pm 0.1$ \\
\hline & & PM & - & $206.2 \pm 21.4$ & $4.9 \pm 0.4$ & $14.3 \pm 1.5$ & - & - & - & - \\
\hline & $\begin{array}{l}\vec{x} \\
\text { 茫 }\end{array}$ & DDM & all & $128.3 \pm 5.9$ & $2.0 \pm 0.1$ & $2.9 \pm 0.1$ & $73.1 \pm 2.7$ & $5588.0 \pm 208.8$ & $2.6 \pm 0.1$ & $4.3 \pm 0.1$ \\
\hline & & $\mathrm{HM}$ & all & $86.6 \pm 5.6$ & $1.5 \pm 0.1$ & $2.3 \pm 0.1$ & $55.6 \pm 2.8$ & $4339.5 \pm 209.8$ & $1.9 \pm 0.1$ & $3.3 \pm 0.1$ \\
\hline \multirow{6}{*}{ 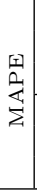 } & & $\mathrm{PM}$ & - & $9.9 \pm 1.1$ & $13.2 \pm 1.0$ & $38.5 \pm 4.0$ & - & - & - & - \\
\hline & $\stackrel{\Xi}{\Xi}$ & DDM & all & $2.9 \pm 0.2$ & $2.8 \pm 0.2$ & $4.3 \pm 0.3$ & $4.2 \pm 0.3$ & $3.9 \pm 0.3$ & $4.4 \pm 0.3$ & $4.4 \pm 0.3$ \\
\hline & & HM & all & $2.4 \pm 0.2$ & $2.3 \pm 0.2$ & $2.7 \pm 0.2$ & $3.0 \pm 0.2$ & $3.0 \pm 0.2$ & $2.9 \pm 0.2$ & $2.8 \pm 0.2$ \\
\hline & & PM & - & $10.1 \pm 1.0$ & $14.1 \pm 1.1$ & $38.5 \pm 4.0$ & - & - & - & - \\
\hline & $\begin{array}{l}\vec{x} \\
\text { 茫 }\end{array}$ & DDM & all & $6.3 \pm 0.3$ & $5.8 \pm 0.3$ & $7.8 \pm 0.3$ & $7.9 \pm 0.3$ & $8.0 \pm 0.3$ & $8.1 \pm 0.3$ & $8.0 \pm 0.3$ \\
\hline & & HM & all & $4.2 \pm 0.3$ & $4.4 \pm 0.3$ & $6.2 \pm 0.3$ & $6.0 \pm 0.3$ & $6.2 \pm 0.3$ & $6.0 \pm 0.3$ & $6.0 \pm 0.3$ \\
\hline \multirow{6}{*}{$\begin{array}{l}0 \\
0 \\
0 \\
\sum_{1} \\
D_{1}\end{array}$} & \multirow{3}{*}{$\stackrel{\vec{\Xi}}{ }$} & PM & - & $0.76 \pm 0.02$ & $0.52 \pm 0.02$ & $0.12 \pm 0.02$ & - & - & - & - \\
\hline & & DDM & all & $0.99 \pm 0.01$ & $0.99 \pm 0.01$ & $0.99 \pm 0.01$ & $0.99 \pm 0.01$ & $0.98 \pm 0.01$ & $0.99 \pm 0.01$ & $0.98 \pm 0.01$ \\
\hline & & $\mathrm{HM}$ & all & $0.99 \pm 0.01$ & $0.99 \pm 0.01$ & $0.99 \pm 0.01$ & $0.99 \pm 0.01$ & $0.98 \pm 0.01$ & $0.99 \pm 0.01$ & $0.98 \pm 0.01$ \\
\hline & \multirow{3}{*}{ 䒝 } & $\mathrm{PM}$ & - & $0.76 \pm 0.01$ & $0.39 \pm 0.06$ & $0.12 \pm 0.05$ & - & - & - & - \\
\hline & & DDM & all & $0.99 \pm 0.01$ & $0.99 \pm 0.01$ & $0.99 \pm 0.01$ & $0.99 \pm 0.01$ & $0.98 \pm 0.01$ & $0.99 \pm 0.01$ & $0.98 \pm 0.01$ \\
\hline & & $\mathrm{HM}$ & all & $0.99 \pm 0.01$ & $0.99 \pm 0.01$ & $0.99 \pm 0.01$ & $0.99 \pm 0.01$ & $0.98 \pm 0.01$ & $0.99 \pm 0.01$ & $0.98 \pm 0.01$ \\
\hline
\end{tabular}

Table 8: Comparison between the best PM, DDM, and HM in predicting the different parameters of NSP2 according to Table 6. Table reports the errors measured with the MAE, MAPE, and PPMCC (see Subsection 3.1) in the interpolation and extrapolation scenarios with best FS according to Table 6 for the different parameters of NSP2 (see Table 4). Note that for the PM the best FS is not indicated since it always uses just a subset of the FS1 and that the PM is only able to predict a subset of the parameters of NSP2 (see Subsection 3.2.

\begin{tabular}{|c|c|c|c|c|c|c|c|c|c|}
\hline & & & FS & $f_{\mathrm{c}}$ & $\mathrm{RNL}_{\mathrm{c}}$ & $\mathrm{RNL}_{\mathrm{b}}$ & $\alpha$ & $\beta$ & $\gamma$ \\
\hline \multirow{6}{*}{ 岳 } & & $\mathrm{PM}$ & - & $202.0 \pm 21.5$ & $4.6 \pm 0.3$ & $14.3 \pm 1.5$ & - & - & - \\
\hline & 节 & DDM & all & $60.3 \pm 4.6$ & $1.0 \pm 0.1$ & $1.6 \pm 0.1$ & $2.4 \pm 0.1$ & $3.0 \pm 0.1$ & $0.5 \pm 0.0$ \\
\hline & & $\mathrm{HM}$ & all & $49.2 \pm 3.4$ & $0.8 \pm 0.1$ & $1.1 \pm 0.1$ & $1.7 \pm 0.1$ & $2.0 \pm 0.1$ & $0.3 \pm 0.0$ \\
\hline & & $\mathrm{PM}$ & - & $206.2 \pm 21.4$ & $4.9 \pm 0.4$ & $14.3 \pm 1.5$ & - & - & - \\
\hline & $x$ & DDM & all & $127.2 \pm 6.0$ & $2.1 \pm 0.1$ & $3.0 \pm 0.1$ & $3.9 \pm 0.1$ & $4.7 \pm 0.1$ & $0.8 \pm 0.0$ \\
\hline & & $\mathrm{HM}$ & all & $90.3 \pm 5.2$ & $1.5 \pm 0.1$ & $2.2 \pm 0.1$ & $3.1 \pm 0.1$ & $3.8 \pm 0.1$ & $0.6 \pm 0.0$ \\
\hline \multirow{6}{*}{ 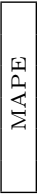 } & & $\mathrm{PM}$ & - & $9.9 \pm 1.1$ & $13.2 \pm 1.0$ & $38.5 \pm 4.0$ & - & - & - \\
\hline & $\stackrel{\vec{z}}{\Xi}$ & DDM & all & $3.0 \pm 0.2$ & $2.8 \pm 0.2$ & $4.2 \pm 0.3$ & $5.9 \pm 0.3$ & $6.2 \pm 0.3$ & $6.1 \pm 0.3$ \\
\hline & & $\mathrm{HM}$ & all & $2.4 \pm 0.2$ & $2.4 \pm 0.2$ & $2.9 \pm 0.2$ & $4.2 \pm 0.3$ & $4.3 \pm 0.3$ & $4.0 \pm 0.3$ \\
\hline & & PM & 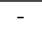 & $10.1 \pm 1.0$ & $14.1 \pm 1.1$ & $38.5 \pm 4.0$ & - & - & - \\
\hline & \begin{tabular}{|l}
$\vec{x}$ \\
$x$ \\
$x$
\end{tabular} & DDM & all & $6.2 \pm 0.3$ & $6.1 \pm 0.3$ & $8.2 \pm 0.3$ & $9.9 \pm 0.3$ & $9.9 \pm 0.3$ & $9.8 \pm 0.3$ \\
\hline & & $\mathrm{HM}$ & all & $4.4 \pm 0.3$ & $4.2 \pm 0.3$ & $5.9 \pm 0.3$ & $7.7 \pm 0.3$ & $8.1 \pm 0.3$ & $8.0 \pm 0.3$ \\
\hline \multirow{6}{*}{$\mid \begin{array}{l}0 \\
0 \\
\sum_{\lambda} \\
0 \\
D\end{array}$} & \multirow{3}{*}{ 节 } & PM & - & $0.76 \pm 0.03$ & $0.52 \pm 0.01$ & $0.12 \pm 0.07$ & - & - & - \\
\hline & & DDM & all & $0.99 \pm 0.01$ & $0.99 \pm 0.01$ & $0.99 \pm 0.01$ & $0.99 \pm 0.01$ & $0.99 \pm 0.01$ & $0.98 \pm 0.01$ \\
\hline & & $\mathrm{HM}$ & all & $0.99 \pm 0.01$ & $0.99 \pm 0.01$ & $0.99 \pm 0.01$ & $0.99 \pm 0.01$ & $0.99 \pm 0.01$ & $0.98 \pm 0.01$ \\
\hline & \multirow{3}{*}{ 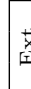 } & $\mathrm{PM}$ & - & $0.76 \pm 0.05$ & $0.39 \pm 0.01$ & $0.12 \pm 0.14$ & - & - & - \\
\hline & & DDM & all & $0.99 \pm 0.01$ & $0.99 \pm 0.01$ & $0.99 \pm 0.01$ & $0.99 \pm 0.01$ & $0.99 \pm 0.01$ & $0.99 \pm 0.01$ \\
\hline & & $\mathrm{HM}$ & all & $0.99 \pm 0.01$ & $0.99 \pm 0.01$ & $0.99 \pm 0.01$ & $0.99 \pm 0.01$ & $0.99 \pm 0.01$ & $0.98 \pm 0.01$ \\
\hline
\end{tabular}

Table 9: Comparison between the best PM, DDM, and HM in predicting the different parameters of NSP3 according to Table 6. Table reports the errors measured with the MAE, MAPE, and PPMCC (see Subsection 3.1) in the interpolation and extrapolation scenarios with best FS according to Table 6 for the different parameters of NSP3 (see Table 4. Note that for the PM the best FS is not indicated since it always uses just a subset of the FS1 and that the PM is only able to predict a subset of the parameters of NSP2 (see Subsection 3.2. 

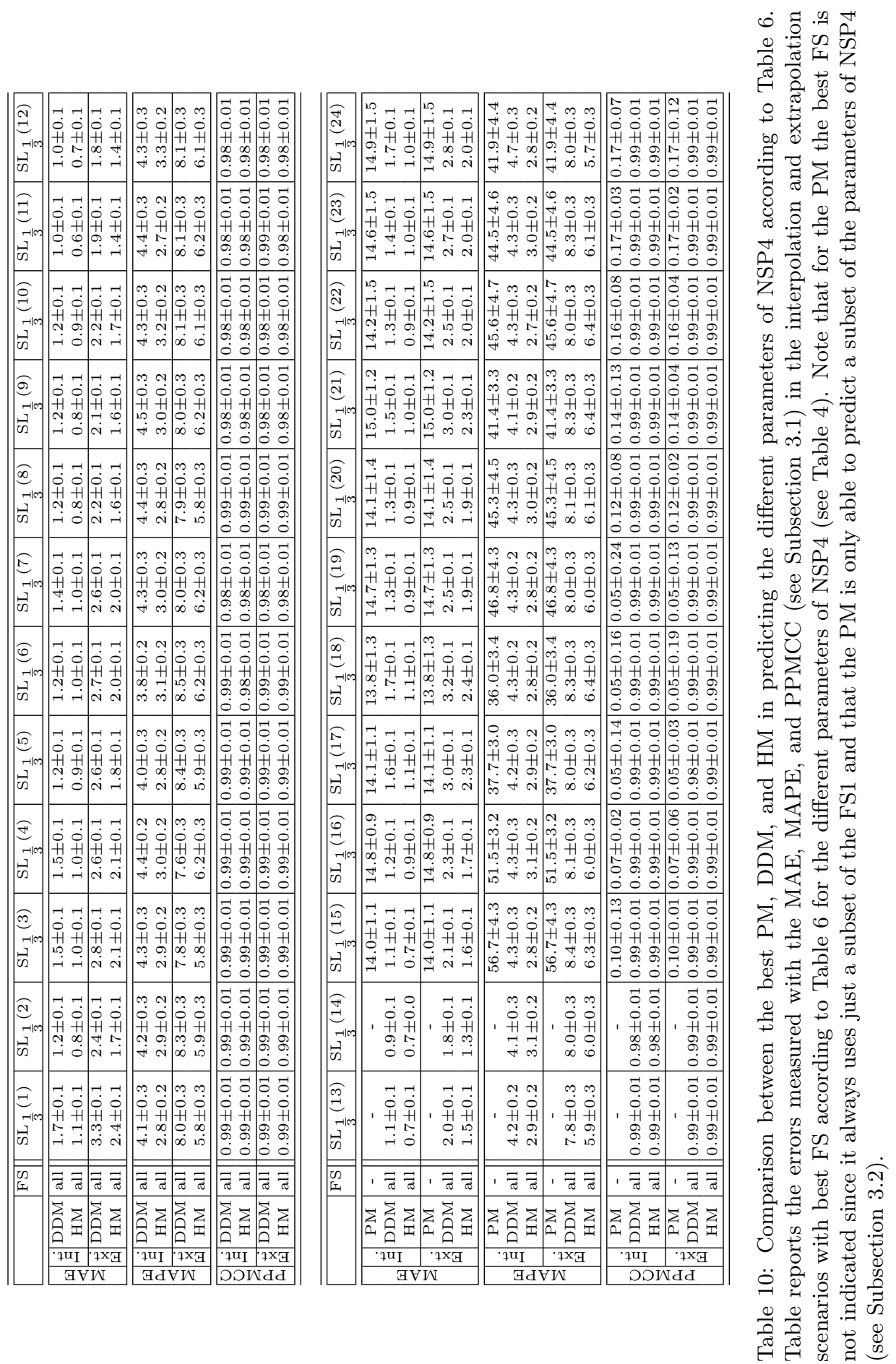

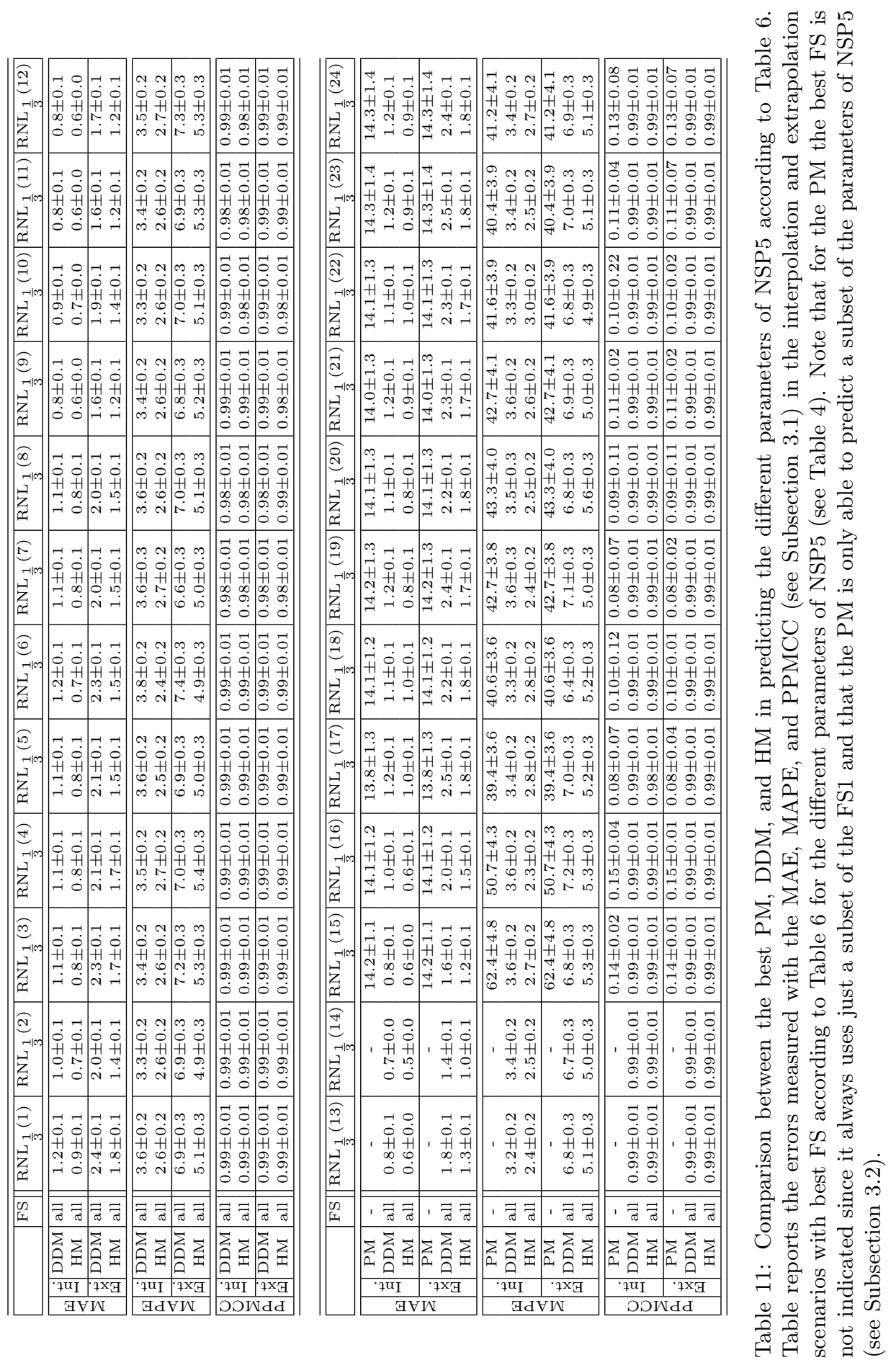


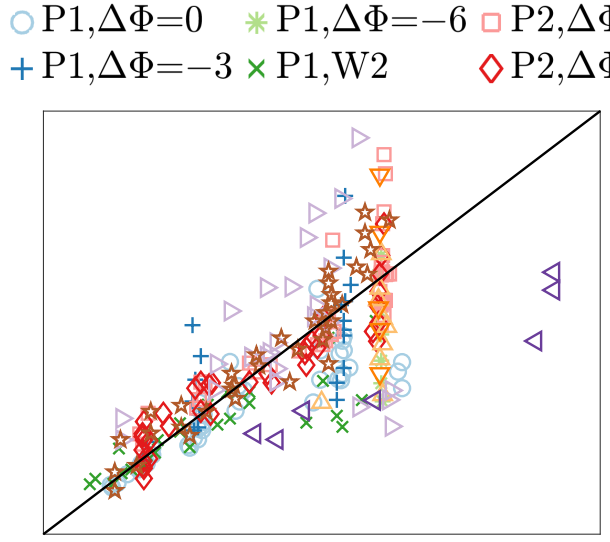

(a) $f_{\mathrm{c}}, \mathrm{PM}$

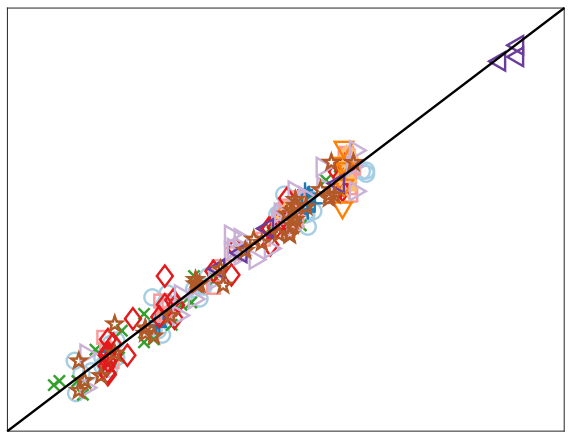

(c) $f_{\mathrm{c}}$, DDM, FSall

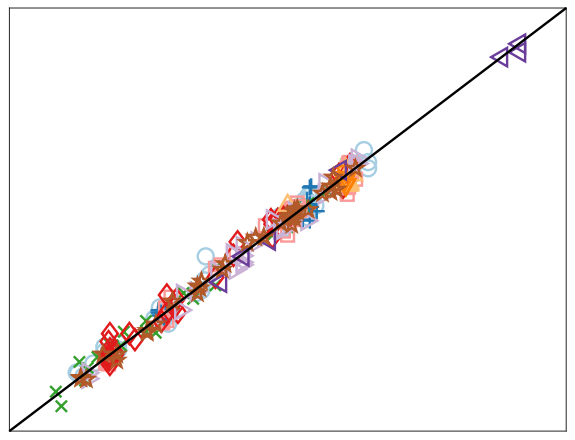

(e) $f_{\mathrm{c}}$, HM, FSall $\triangle \mathrm{P} 2, \Delta \Phi=-3 \triangleright \mathrm{P} 3, \mathrm{~W} 4$ « $\mathrm{P} 3, \mathrm{~W} 6$ $2 \nabla \mathrm{P} 2, \Delta \Phi=-5 \triangleleft \mathrm{P} 3, \mathrm{~W} 5$

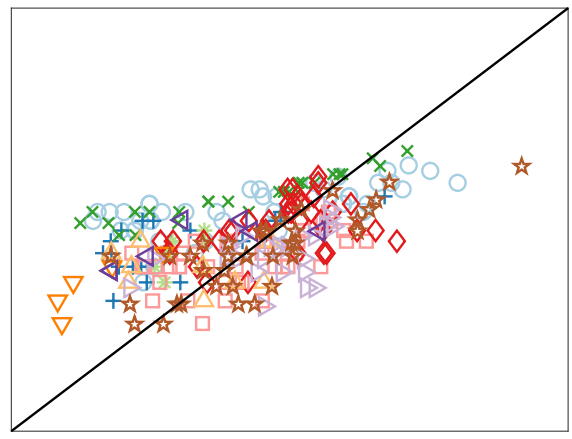

(b) $\mathrm{RNL}_{\mathrm{c}}, \mathrm{PM}$

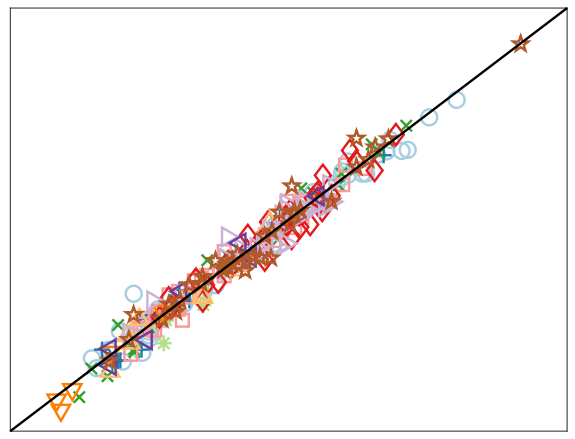

(d) $\mathrm{RNL}_{\mathrm{c}}$, DDM, FSall

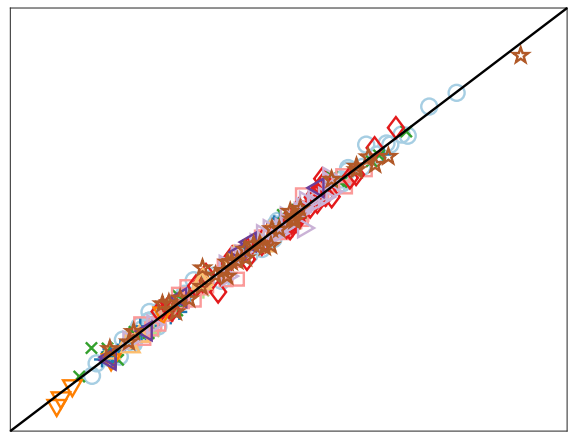

(f) $\mathrm{RNL}_{\mathrm{c}}, \mathrm{HM}$, FSall

Figure 22: Comparison between the best PM, DDM, and HM in predicting the different parameters of NSP1 according to Table6. Figure reports the scatter plot (measured values on the $\mathrm{x}$ axis and predicted ones on the $\mathrm{y}$ axis) in the interpolation scenario with best FS according to Table 6 for the different parameters of NSP1 (see Table 4 . 


\section{$\circ \mathrm{G} 1+\mathrm{G} 2 * \mathrm{G} 3 \times \mathrm{G} 4 \square \mathrm{G} 5 \diamond \mathrm{G} 6$}

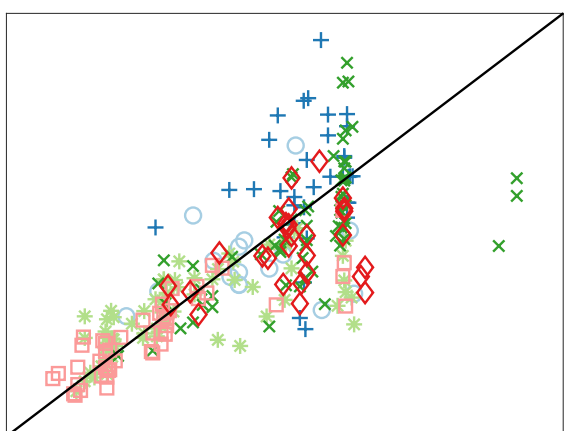

(a) $f_{\mathrm{c}}, \mathrm{PM}$

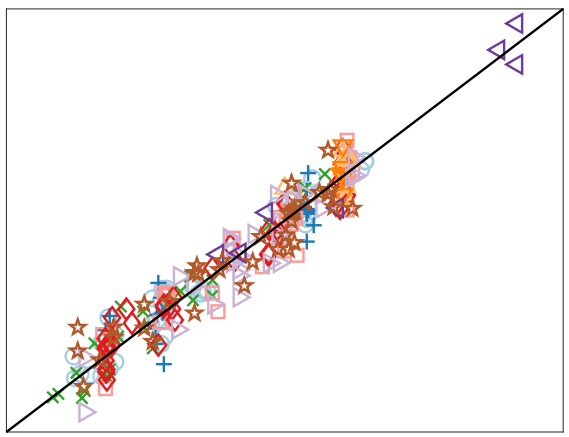

(c) $f_{\mathrm{c}}, \mathrm{DDM}$, FSall

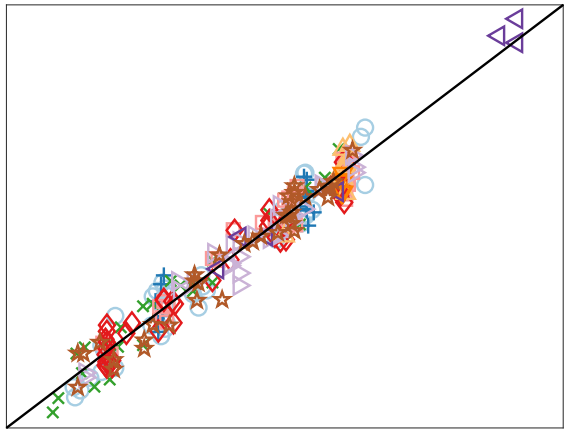

(e) $f_{\mathrm{c}}, \mathrm{HM}$, FSall

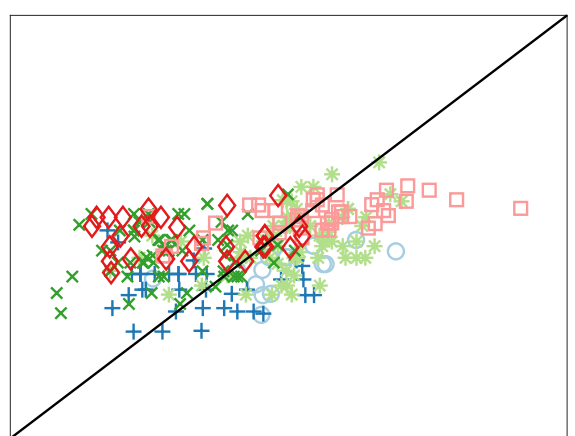

(b) $\mathrm{RNL}_{\mathrm{c}}, \mathrm{PM}$

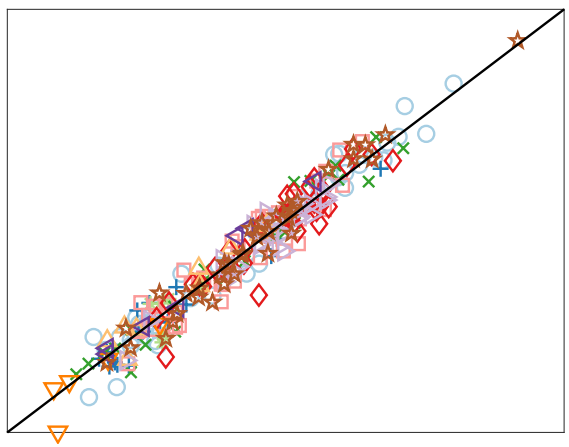

(d) $\mathrm{RNL}_{\mathrm{c}}, \mathrm{DDM}$, FSall

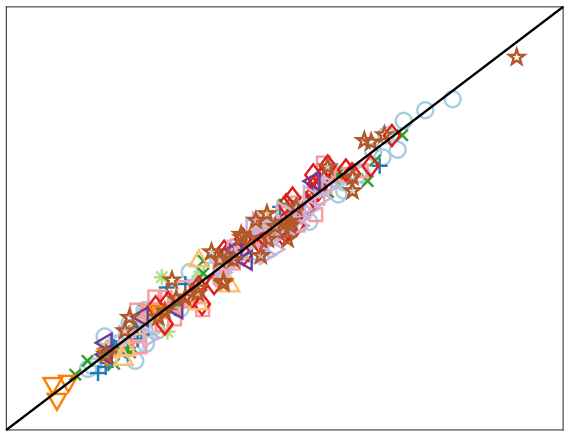

(f) $\mathrm{RNL}_{\mathrm{c}}, \mathrm{HM}$, FSall

Figure 23: Comparison between the best PM, DDM, and HM in predicting the different parameters of NSP1 according to Table 6. Figure reports the scatter plot (measured values on the $\mathrm{x}$ axis and predicted ones on the $\mathrm{y}$ axis) in the extrapolation scenario with best FS according to Table 6 for the different parameters of NSP1 (see Table 4). 
the cavitation phenomenon. Pure data-driven models, built on experimental data, exploiting the most advanced deep learning based tools since domain of the resulting feature is characterised by a relatively high cardinality, if compared with the sample size, and then conventional data driven models were proven to be inadequate. Finally, hybrid models, exploiting both the physical knowledge of the problem and the experimental data.

Results obtained with the different modelling strategies in two different scenarios have been compared, allowing formulating interesting conclusions:

- the performance of the proposed approach in the tested scenarios are remarkable, confirming the effectiveness of the proposed methods;

- only exploiting deep learning bases methodologies it is possible to exploit the rich information enclosed in the more complex features, like the detailed flow characterisation obtainable by calculations carried out with a Boundary Element Method;

- among the different target definitions considered, the best performances are obtained with the one-third octave band spectra (NSP4 and NSP5 in the paper), however differences in accuracies are in general rather small;

- among the different feature sets considered, the best performances are obtained when all the features are considered together, and the relative trends of the errors confirm the possibility to improve the performance of the modelling approach by considering detailed flow quantities. The effects of the different feature sets, even if not remarkable in terms of absolute values, are still quite noticeable since the errors are, in some cases, improved more than $50 \%$;

- the errors of all the considered models are almost doubled when passing from the interpolation to the extrapolation scenario. Nevertheless, in practical application, they are still satisfactory;

- the effectiveness of the physical models in modelling the complex behaviour of cavitation noise is limited, however, the physical knowledge encapsulated in them allows improving the performance of the pure data driven approach, confirming the suitability of the hybrid approach for this kind of problems.

In summary, according to these results, the proposed approach is definitely promising, however its potential needs to be further investigated. In particular, the inclusion of detailed flow quantity, although improving the performance of the method in a relative sense, has a somewhat limited effect from a practical point of view. This fact may be partially ascribed to the fact that also simpler modelisation approaches allowed obtaining good results in the tested scenario. It must be noticed that, despite the effort 
spent in building the dataset, it still accounts for a rather limited number of propellers and configurations, preventing the opportunity to verify the performance of the method on fully unseen cases.

Future activities will be therefore dedicated to the enlargement of the dataset and to the investigation of more realistic scenarios, as the prediction of noise for a completely unseen propeller and configuration.

\section{Acknowledgements}

The authors wish to express their gratitude to Fincantieri MM-ARC office, which kindly provided the propeller models used in the present work.

\section{References}

[1] D. Ross, Mechanics of Underwater Noise, Peninsula Publishing, 1976.

[2] Council of European Union, Directive 2008/56/EC, https://eur-lex.europa.eu/legal-content/EN/TXT/?uri=CELEX : 32008L0056 (2008).

[3] IMO, Resolution MSC. 337(91), Code on Noise Levels on Board Ships, London (2012).

[4] D. Bertetta, S. Brizzolara, S. Gaggero, M. Viviani, L. Savio, Cpp propeller cavitation and noise optimization at different pitches with panel code and validation by cavitation tunnel measurements, Ocean engineering 53 (2012) 177-195.

[5] S. Gaggero, G. Tani, D. Villa, M. Viviani, P. Ausonio, P. Travi, G. Bizzarri, F. Serra, Efficient and multi-objective cavitating propeller optimization: An application to a high-speed craft, Applied Ocean Research 64 (2017) 31-57.

[6] J. Bosschers, Investigation of hull pressure fluctuations generated by cavitating vortices, in: International symposium on marine propulsors, 2009.

[7] A. E. Raestad, Tip vortex index-an engineering approach to propeller noise prediction, The Naval Architect (1996) 11-15.

[8] J. Bosschers, A semi-empirical prediction method for broadband hullpressure fluctuations and underwater radiated noise by propeller tip 
vortex cavitation, Journal of Marine Science and Engineering 6 (2) (2018) 49 .

[9] N. A. Brown, Thruster noise, in: Dynamic Positioning Conference, 1999.

[10] F. H. Lafeber, J. Bosschers, Validation of computational and experimental prediction methods for the underwater radiated noise of a small research vessel, in: International Symposium on Ships and Other Floating Structures, 2016.

[11] J. P. Petersen, O. Winther, D. J. Jacobsen, A machine-learning approach to predict main energy consumption under realistic operational conditions, Ship Technology Research 59 (1) (2012) 64-72.

[12] T. W. P. Smith, E. O'Keeffe, L. Aldous, P. Agnolucci, Assessment of shipping's efficiency using satellite ais data, in: International Council on Clean Transportation, 2013.

[13] A. Coraddu, L. Oneto, F. Baldi, D. Anguita, Vessels fuel consumption forecast and trim optimisation: a data analytics perspective, Ocean Engineering 130 (2017) 351-370.

[14] F. Cipollini, L. Oneto, A. Coraddu, A. J. Murphy, D. Anguita, Condition-based maintenance of naval propulsion systems: Data analysis with minimal feedback, Reliability Engineering \& System Safety 177 (2018) 12-23.

[15] D. Calcagni, G. Bernardini, F. Salvatore, Automated marine propeller optimal design combining hydrodynamics models and neural networks, in: International Conference on Computer Applications and Information Technology in the Maritime Industries, 2012.

[16] H. Yang, S. Shen, X. Yao, M. Sheng, C. Wang, Competitive deep-belief networks for underwater acoustic target recognition, Sensors 18 (4) (2018) 952 .

[17] W. Soares-Filho, J. M. de Seixas, L. P. Caloba, Principal component analysis for classifying passive sonar signals, in: IEEE International Symposium on Circuits and Systems, 2001.

[18] B. Aktas, A systematic experimental approach to cavitation noise prediction of marine propellers, Ph.D. thesis, Newcastle University (2017). 
[19] L. Miglianti, F. Cipollini, L. Oneto, G. Tani, M. Viviani, Model scale cavitation noise spectra prediction: Combining physical knowledge with data science, Ocean Engineering 178 (2019) 185-203.

[20] S. Gaggero, D. Villa, S. Brizzolara, Rans and panel method for unsteady flow propeller analysis, Journal of Hydrodynamics, Ser. B 22 (5) (2010) $564-569$.

[21] S. Gaggero, D. Villa, M. Viviani, An investigation on the discrepancies between ranse and bem approaches for the prediction of marine propeller unsteady performances in strongly non-homogeneous wakes, in: International Conference on Ocean, Offshore and Arctic Engineering, 2014 .

[22] S. Gaggero, D. Villa, Cavitating propeller performance in inclined shaft conditions with openfoam: Pptc 2015 test case, Journal of Marine Science and Application 17 (1) (2018) 1-20.

[23] I. Goodfellow, Y. Bengio, A. Courville, Deep learning, MIT press, 2016.

[24] ITTC Specialist Committee on Hydrodynamic Noise, Model-scale propeller cavitation noise measurements, Recommended Procedures and Guidelines 7. 5-02-01-05, International Towing Tank Conference (2017).

[25] G. Tani, B. Aktas, M. Viviani, M. Atlar, Two medium size cavitation tunnel hydro-acoustic benchmark experiment comparisons as part of a round robin test campaign, Ocean Engineering 138 (2017) 179-207.

[26] ITTC Propulsion Committee , Model-scale cavitation tests, Recommended Procedures and Guidelines 7. 5-02-03-03. 1, International Towing Tank Conference (2017).

[27] P. Welch, The use of fast fourier transform for the estimation of power spectra: a method based on time averaging over short, modified periodograms, IEEE Transactions on audio and electroacoustics 15 (2) (1967) 70-73.

[28] G. Tani, M. Viviani, M. Ferrando, E. Armelloni, Aspects of the measurement of the acoustic transfer function in a cavitation tunnel, Applied Ocean Research 87 (2019) 264-278.

[29] G. Tani, M. Viviani, E. Armelloni, M. Nataletti, Cavitation tunnel acoustic characterisation and application to model propeller radiated 
noise measurements at different functioning conditions, Proceedings of the Institution of Mechanical Engineers, Part M: Journal of Engineering for the Maritime Environment 230 (2) (2016) 250-266.

[30] A. Y. Odabaşi, P. A. Fitzsimmons, Alternative methods for wake quality assessment, International Shipbuilding Progress 25 (282) (1978) 3442.

[31] A. M. Keller, Enige aspecten bij het ontwerpen van scheepsschroeven, Publicatie in: Schip en Werf, 33e Jaargang, No. 24, blz. 658-663 (1966).

[32] L. C. Burrill, A. Emerson, Propeller cavitation: Further tests on 16in. propeller models in the king's college cavitation tunnel, International Shipbuilding Progress 10 (104) (1963) 119-131.

[33] J. Kerwin, S. Kinnas, J. Lee, W. Shih, A surface panel method for the hydrodynamic analysis of ducted propellers, Tech. rep., Massachusetts Inst of Tech Cambridge Dept of Ocean Engineering (1987).

[34] S. Kinnas, C. Hsin, Boundary element method for the analysis of the unsteady flow aroundextreme propeller geometries, AIAA journal 30 (3) (1992) 688-696.

[35] S. Kinnas, N. Fine, A numerical nonlinear analysis of the flow around two-and three-dimensional partially cavitating hydrofoils, Journal of Fluid Mechanics 254 (1993) 151-181.

[36] S. Kinnas, N. Fine, A nonlinear boundary element method for the analysis of unsteady propeller sheet cavitation, in: Nineteenth Symposium on Naval Hydrodynamics, 1992.

[37] Y. Young, S. Kinnas, A bem for the prediction of unsteady midchord face and/or back propeller cavitation, J. Fluids Eng. 123 (2) (2001) $311-319$.

[38] H. Lee, S. Kinnas, Application of a boundary element method in the prediction of unsteady blade sheet and developed tip vortex cavitation on marine propellers, Journal of Ship Research 48 (1) (2004) 15-30.

[39] Y. Young, S. Kinnas, Numerical modeling of supercavitating propeller flows, Journal of Ship Research 47 (1) (2003) 48-62.

[40] B. Gindroz, T. Hoshino, J. V. Pylkkanen, Ittc propulsion committee propeller rans/panel method workshop, in: International Towing Tank Conference, 1998. 
[41] G. Vaz, J. Bosschers, Validation of a bem for modelling steady sheet cavitation on marine propellers, in: International Conference on Computational Methods in Marine Engineering, 2005.

[42] G. Vaz, J. Bosschers, Modelling three dimensional sheet cavitation on marine propellers using a boundary element method, in: Sixth International Symposium on Cavitation, CAV2006, 2006.

[43] S. Brizzolara, D. Villa, S. Gaggero, A systematic comparison between rans and panel methods for propeller analysis, in: Proceedings of the 8th International Conference on Hydrodynamics, 2008.

[44] S. Gaggero, G. Dubbioso, D. Villa, R. Muscari, M. Viviani, Propeller modeling approaches for off-design operative conditions, Ocean Engineering 178 (2019) 283-305.

[45] S. Gaggero, D. Villa, Steady cavitating propeller performance by using openfoam, starccm+and a boundary element method, Proceedings of the Institution of Mechanical Engineers Part M: Journal of Engineering for the Maritime Environment 231 (2) (2017) 411-440.

[46] S. Gaggero, S. Brizzolara, A panel method for trans-cavitating marine propellers (2009).

[47] S. Gaggero, M. Viviani, G. Tani, F. Conti, P. Becchi, F. Valdenazzi, Comparison of different approaches for the design and analysis of ducted propellers, in: Proceedings of the 5th international conference on computational methods in marine engineering, MARINE2013, 2013.

[48] S. Gaggero, J. Gonzalez-Adalid, M. Sobrino, Design of contracted and tip loaded propellers by using boundary element methods and optimization algorithms, Applied Ocean Research 55 (2016) 102-129.

[49] S. Gaggero, Numerical design of a rim-driven thruster using a ransbased optimization approach, Applied Ocean Research 94 (2020) 101941.

[50] B. Maines, R. E. A. Arndt, The case of the singing vortex, Journal of fluids engineering 119 (2) (1997) 271-276.

[51] F. Proctor, N. Ahmad, G. Switzer, F. Limon Duparcmeur, Threephased wake vortex decay, in: AIAA Atmospheric and Space Environments Conference, 2010. 
[52] S. D. Jessup, An experimental investigation of viscous aspects of propeller blade flow, Ph.D. thesis, Catholic University of America (1989).

[53] Y. T. Shen, S. Gowing, S. D. Jessup, Tip vortex cavitation inception scaling for high reynolds number applications, Journal of Fluids Engineering 131 (7) (2009).

[54] J. Bosschers, An analytical and semi-empirical model for the viscous flow around a vortex cavity, International Journal of Multiphase Flow 105 (2018) 122-133.

[55] T. Hommes, J. Bosschers, H. W. M. Hoeijmakers, Evaluation of the radial pressure distribution of vortex models and comparison with experimental data, Journal of Physics: Conference Series 656 (2015) 012182.

[56] S. Shalev-Shwartz, S. Ben-David, Understanding machine learning: From theory to algorithms, Cambridge university press, 2014.

[57] J. Shawe-Taylor, N. Cristianini, Kernel methods for pattern analysis, Cambridge university press, 2004.

[58] I. Guyon, A. Elisseeff, An introduction to variable and feature selection, The Journal of Machine Learning Research 3 (2003) 1157-1182.

[59] L. Oneto, Model Selection and Error Estimation in a Nutshell, Springer, 2020 .

[60] S. Boyd, L. Vandenberghe, Convex optimization, Cambridge university press, 2004.

[61] P. M. Pardalos, H. E. Romeijn, Handbook of global optimization, Vol. 2, Springer Science \& Business Media, 2013.

[62] V. N. Vapnik, Statistical learning theory, Wiley New York, 1998.

[63] L. L. C. Kasun, H. Zhou, G. B. Huang, C. M. Vong, Representational learning with extreme learning machine for big data, IEEE intelligent systems 28 (6) (2013) 31-34.

[64] J. Tang, C. Deng, G. Huang, Extreme learning machine for multilayer perceptron, IEEE transactions on neural networks and learning systems 27 (4) (2016) 809-821. 
[65] R. Tibshirani, Regression shrinkage and selection via the lasso, Journal of the Royal Statistical Society: Series B (Methodological) 58 (1) (1996) $267-288$.

[66] H. Zou, T. Hastie, Regularization and variable selection via the elastic net, Journal of the royal statistical society: series B (statistical methodology) 67 (2) (2005) 301-320.

[67] J. C. Russ, The image processing handbook, CRC press, 2016.

[68] C. Szegedy, S. Ioffe, V. Vanhoucke, A. A. Alemi, Inception-v4, inception-resnet and the impact of residual connections on learning, in: AAAI Conference on Artificial Intelligence, 2017.

[69] K. He, X. Zhang, S. Ren, J. Sun, Delving deep into rectifiers: Surpassing human-level performance on imagenet classification, in: IEEE international conference on computer vision, 2015.

[70] A. Coraddu, M. Kalikatzarakis, L. Oneto, G. J. Meijn, M. Godjevac, R. D. Geertsmad, Ship diesel engine performance modelling with combined physical and machine learning approach, in: International Naval Engineering Conference and Exhibition, 2018.

[71] L. Coraddu, A. Oneto, F. Baldi, D. Anguita, Vessels fuel consumption forecast and trim optimisation: a data analytics perspective, Ocean Engineering 130 (2017) 351-370. 